Credit Market Distortions, Asset Prices and Monetary Policy

Damjan Pfajfar and Emiliano Santoro

June 2008

CWPE 0825 


\title{
Credit Market Distortions, Asset Prices and Monetary Policy*
}

\author{
DAMJAN PFAJFAR ${ }^{\dagger}$ \\ University of CAMBRidge
}

\author{
EMILIANO SANTORO \\ University of CAMbridge
}

April 7, 2008

\begin{abstract}
In this paper we develop a sticky price DSGE model to study the role of capital market imperfections for monetary policy implementation. Recent empirical and theoretical studies have stressed the effect of firms' external finance on their pricing decisions. The so-called cost channel of the transmission mechanism has been explored within New Keynesian frameworks that pose particular emphasis on inflation dynamics. These models generally disregard the role of external finance for the dynamics of asset prices. We ask whether monetary policy should respond to deviations of asset prices from their frictionless level and, more importantly, if the answer to this question changes when financial frictions are properly taken into account. We analyze these issues from the vantage of equilibrium determinacy and stability under adaptive learning. We show that usual conditions for equilibrium uniqueness and E-stability are significantly altered when the cost channel matters. Nevertheless, we find that responding to actual or expected asset price misalignments helps at restoring determinacy and stability under learning. These conclusions are further enforced in the presence of a high degree of pass-through from policy to bank lending rates.
\end{abstract}

JEL: E31; E32; E52

Keywords: Monetary Policy, Capital Market Imperfections, Cost Channel, Asset Prices.

\footnotetext{
${ }^{*}$ We would like to thank Sean Holly and Seppo Honkapohja for guidance and helpful discussions. We would also like to thank Chryssi Giannitsarou, Refet Gürkaynak, Bruce McGough, Hashem Pesaran, Roberto Tamborini, and other participants in seminars at the Aarhus University, Bilkent University, Cambridge University, Bank of Canada, Cardiff Business School, Koç University, Banco de Portugal, Tilburg University, Uppsala University, and at Catholic University in Milan for their comments. All remaining errors are ours.

${ }^{\dagger}$ Faculty of Economics, Austin Robinson Building, Sidgwick Avenue, Cambridge CB3 9DD, UK. E-mail: dp316@cam.ac.uk.Web: http://www.econ.cam.ac.uk/phd/dp316/.

${ }^{\ddagger}$ Faculty of Economics, Austin Robinson Building, Sidgwick Avenue, Cambridge CB3 9DD, UK. E-mail: es356@cam.ac.uk. Web: http://www.econ.cam.ac.uk/phd/es356/.
} 


\section{INTRODUCTION}

The last two decades have been marked by an increasing occurrence of financial instability episodes. These have often resulted in macroeconomic turmoil, both in industrialized economies and emerging markets. The significance of real costs of financial instability emphasizes the need to explore the link between financial volatility and the real economy. Through its influence on the cost for external finance, the banking sector plays a prominent role in the monetary policy transmission mechanism, determining the amplitude of monetary policy innovations on prices and on firms' profitability. Therefore, it is of crucial importance to account for the effects of bank lending on production, inflation and asset prices. This leads to two central questions: firstly, should the monetary authority respond to asset prices to enhance financial stability and to rule out possible multiple equilibria? Secondly, does the answer to this question depend on whether financial frictions and the banking sector are properly taken into account?

We address these questions within a New Keynesian (NK) framework featuring the existence of a cost channel effect, where firms' marginal cost directly depends on the nominal rate of interest. In recent years, a growing body of cross-country evidence has stressed the relevance of the cost of external finance for pricing decisions of firms. The literature on the cost channel of monetary policy transmission mechanism has placed particular emphasis on the role of supplyside effects for inflation dynamics. Ravenna and Walsh (2006) and Chowdhury, Hoffmann, and Schabert (2006) explore the cost channel in a model with nominal rigidities. They show that the traditional output-inflation trade-off can be significantly modified, as the cost channel substantially alters the transmission of monetary policy. Despite the increasing emphasis placed on financial frictions as an amplification mechanism of macroeconomic fluctuations (see Bernanke and Gertler, 1989), the effect of the cost channel on firms' profitability and on asset prices has generally been neglected by the literature exploring the normative aspects of monetary policy.

Supply-side effects exerted by nominal interest rates can be interpreted as a direct consequence of capital market imperfections, namely asymmetric information giving rise to agency problems between firms and lenders. The magnitude of monetary policy supply-side effects not only relates to firms' dependence on external funds but also on the pass-through from policy to bank lending rates. This aspect is central to our analysis. Chowdhury, Hoffmann, and Schabert (2006) show that heterogeneous financial systems can lead to major differences in the transmission of policy shocks. In the perspective of designing the architecture of an optimal currency area, convergence of financial systems seems to be an important prerequisite for a successful common monetary policy. These considerations turn out to be particularly relevant for the European Monetary Union (EMU), where a single monetary authority influences heterogeneous credit markets.

The present work builds on the theoretical literature on fragility and instability fostered by imperfect financial markets. These studies have often produced models of interaction between financial and goods markets. As detailed in Greenwald and Stiglitz (1988, 1993), Bernanke and Gertler $(1989,1990)$ and Kiyotaki and Moore (1997), financing constraints can be relevant to both investment and production decisions. In our framework, firms are committed to pay 
the wage bill before production takes place and profits are realized. Although firms could alternatively issue new equity, this possibility is a priori excluded. In fact, due to adverse selection phenomena, new equity issues would be too costly. Therefore, firms have to resort to the credit market. Asymmetric information only characterizes the equity market, whereas the banking sector is assumed to have perfect information.

We study the performance of interest rate rules responding to (current or expected) inflation, output gap and asset prices misalignments from their frictionless level when the cost channel matters. We tackle the problem from the vantage of rational expectations equilibrium (REE) uniqueness and stability under adaptive learning. As a matter of fact, Brückner and Schabert (2003) and Llosa and Tuesta (2007) show that the cost channel significantly modifies standard conditions for determinacy and learnability within the context of a NK model. However, they only consider a one-to-one relationship between the monetary instrument and the bank-lending rate.

This study considers the entire range of pass-through from policy to bank lending rates. We provide an analytical assessment of the conditions for determinacy and E-stability under an interest rate rule that responds only to inflation. In this case, we provide critical values of the degree of pass-through that significantly alter the standard properties of the NK model in terms of equilibrium uniqueness and expectational stability. As it is well known, interest rate rules usually ensure a unique equilibrium if they fulfill the so-called Taylor principle. We show that this condition is no longer sufficient to ensure determinacy when the cost channel matters, as we identify a set of stricter conditions that bound the response to the rate of inflation from above. Given that the nominal rate of interest has a direct effect on inflation, these constraints prevent the central bank from being too reactive to inflationary pressures. Moreover, we show that considering a one-to-one relationship from policy to bank lending rates, as in Ravenna and Walsh (2006), can be quite misleading for the design of monetary policy. Conversely, a careful assessment of the degree of pass-through is necessary to understand the magnitude of supply-side effects.

Numerical simulations show that the area of determinacy and E-stability considerably decreases in the degree of pass-through. We argue that, under strong credit market distortions, the monetary policy authority should be cautious when designing its policy. A response to either output gap or asset prices is desirable under these circumstances, as this reduces the area of indeterminacy and E-instability. When we compare the two alternatives we observe that in some cases a response to asset prices might be even more attractive than responding to the output gap, as a smaller reaction coefficient is required to drive the system in the area of determinacy.

Moreover, under uncertainty regarding the degree of pass-through between policy and bank lending rates, we suggest that the central bank is better off following a rule in which the policy instrument is set according to misalignments in contemporaneous data rather than adopting forward looking interest rate rules. In the former case a considerably wider area of determinacy and a lower welfare loss is achieved for a plausible range of responses to inflation and output gap. Furthermore, responding to asset prices is always beneficial, from a welfare point of view, 
when the central bank considers a term reflecting stock price volatility in the loss function.

Our results point out that responding to asset prices misalignments might be beneficial when strong credit market distortions are detected. This strategy generally allows us to ensure equilibrium uniqueness and learnability. Due to a direct effect induced by interest rate movements on firms' profitability, these results stand in contrast with previous studies exploring the opportunity to stabilize asset prices in the presence of nominal rigidities. As a matter of fact, Carlstrom and Fuerst (2007) show that the central bank can inadvertently introduce real indeterminacy into the economy by responding positively to asset prices misalignments. They suggest that this is due to the inverse relationship between marginal costs and dividends that classically arises in a sticky price environment. In our case, the interest rate directly affects firms' profitability and, in turn, asset prices. When the cost channel matters, the policy instrument affects positively the marginal cost, hence a monetary innovation exerts a direct influence on inflation dynamics in the same direction. At the same time, inflationary pressures are scaled down if the central bank responds positively to asset prices, as this translates into a negative response to the output gap that balances the cost channel effect.

The remainder of the paper reads as follows: Section 1 reviews some relevant literature; Section 2 introduces the theoretical setting; Section 3 draws some policy implications in a frictionless environment; Section 4 assesses the conditions for determinacy and E-stability under different interest rate rules; Section 5 concludes.

\section{Literature Review}

In principle, the cost channel has been advanced as a possible explanation of a positive reaction of the price level to contractionary monetary policy observed in several empirical studies. Sims (1992) was the first to point out this unexpected finding for the United States, readily labelled by Eichenbaum (1992) as the price puzzle. Numerous studies have focused on the role of the cost channel for the transmission mechanism of monetary policy, both from an empirical and a theoretical perspective. Barth and Ramey (2000) provide evidence in support of the cost channel in industry-level longitudinal data, whereas Christiano, Eichenbaum, and Evans (1997) incorporate the cost channel into an aggregate structural model. Compared to previous studies that primarily focus on banks operating in a frictionless credit market (see, e.g., Ravenna and Walsh, 2006), Hülsewig, Mayer, and Wollmershäuser (2006) assess the cost channel from a different perspective. They conjecture that banks determine their loan supply in light of expectations about the future course of monetary policy. This framework implies that the adjustment of loan rates to a monetary policy shock is sticky. Chowdhury, Hoffmann, and Schabert (2006) estimate different Phillips curves that account for direct interest rate effects. They show that changes in short-run nominal interest rates have a substantial direct effect on inflation dynamics in the majority of developed countries. Their structural model reveals that the cost channel can substantially dampen inflation responses, and is even able to account for inverse inflation reactions, which can be related to the price puzzle. Thus, their analysis points at significant direct interest rate effects on short-run inflation dynamics, indicating that the cost channel is non-negligible for the assessment of the monetary policy transmission mechanism. 
Nonetheless, the existing literature on the cost channel does not explore the effect of firms' reliance on external finance on their profitability and, in turn, on stock price dynamics. Our paper addresses this issue and evaluates the opportunity for the monetary authority to respond to asset prices misalignments when the cost channel matters. A long-standing debate concerning the role and scope of central banks in stabilizing asset prices has arisen from the contributions of Bernanke and Gertler $(1999,2001)$, where financial frictions give rise to a financial accelerator mechanism that magnifies the effects of both exogenous and policy shocks. In their sticky price framework a shock to asset prices increases aggregate demand, hence driving up the price level. Bernanke and Gertler $(1999,2001)$ conclude that there is no need for a direct response to asset prices, as a central bank that responds to general price inflation is implicitly responding to asset price movements. They argue in favor of a monetary policy that does not respond to asset prices, except insofar as they signal changes in expected inflation. Bullard and Schaling (2002) show that adding equity prices to the Taylor rule generally does not improve the economic performance, and might possibly harm both real and financial stability. ${ }^{1}$ Conversely, Genberg, Lipsky, Cecchetti, and Wadhwani (2000) follow the modelling strategy of Bernanke and Gertler (1999, 2001), and argue that central banks should respond to asset prices to stabilize the economy and to prevent from the rise of bubbles. ${ }^{2}$ Carlstrom and Fuerst (2007) emphasize the link between profitability and output gap in a sticky price environment. They show how a central bank trying to avoid bubbles can inadvertently introduce non-fundamental movements into both asset prices and real activity by reacting to asset prices misalignments. It is a wellestablished fact that in sticky price models marginal costs are proportional to the output gap. An interest rate rule that responds positively to (expected or current) values of stock prices is a rule that responds positively to dividends. This creates a potential problem from the perspective of equilibrium determinacy. Nisticò (2006) and Airaudo, Nisticò, and Zanna (2006) analyze the role of stock prices for monetary policy design in a small structural model with stock-wealth effects. They find that adopting an instrument rule that responds to the stockprice gap incurs risks of endogenous instability that depend on the average price markup in the economy, while reacting to the stock-price growth can achieve substantial stability gains. Faia and Monacelli (2007) study optimal Taylor-type interest rate rules in an economy with credit market imperfections. In their sticky price framework, a countercyclical premium on external finance is generated through a bankruptcy mechanism. They find that monetary policy should lower interest rates in the face of positive misalignments of asset prices from their equilibrium level. Nevertheless, when the monetary authority is strenuously committed to stabilize inflation, responding to asset prices does not bring any benefit from a welfare point of view. Gilchrist and Saito (2006) reinforce previous results that a policy responding strongly to inflation, in absence of financial frictions, is sufficient. Adding asset prices to the set of intermediate targets does not lead to further benefits. Yet, none of these studies considers the opportunity of responding

\footnotetext{
${ }^{1}$ Nevertheless, they implement an arbitrage condition where the dividend process is not modelled as a function of the profits.

${ }^{2}$ Bernanke and Gertler (2001) comment on these results claiming that, although the models used are similar, Genberg, Lipsky, Cecchetti, and Wadhwani (2000) assume that the policymaker knows with certainty the stock price process and, most importantly, when the bubble is going to burst.
} 
to asset prices misalignments when capital market imperfections, in the form of a cost channel, are explicitly taken into account. As a matter of fact, most of the studies that argue against an explicit response to asset prices misalignments, generally disregard the importance of the joint direct effect of the cost of working capital on firms' pricing process and on their profitability. They mainly focus on the role of wealth effects channeled through the demand side.

\section{The Model}

2.1. Demand Side. The model economy is populated by households, firms, and financial intermediaries operating on the markets for consumption goods, labor, assets and bonds. The goods market is characterized by monopolistic competition, and the adjustment of prices follows the standard treatment based on Calvo (1983). Derivations of the basic NK model can be found in Woodford (2003) and Walsh (2003). Preferences of the representative household are defined over a composite consumption good, $C_{t}$ and leisure, $1-N_{t}$. Households maximize the expected present discounted value of their utility:

$$
E_{t} \sum_{i=0}^{\infty} \beta^{i}\left[\frac{H_{t+i} C_{t+i}^{1-\sigma}}{1-\sigma}-\frac{N_{t+i}^{1+\eta}}{1+\eta}\right],
$$

where $\beta$ is the intertemporal discount factor and $H_{t}=\exp \left(h_{t}\right)$ is a taste shock. ${ }^{3}$ Parameter $\sigma$ denotes the inverse of the intertemporal elasticity of substitution, while $\eta$ is the inverse of the elasticity of substitution between work and leisure. Consumption, $C_{t}$, is a Dixit-Stiglitz bundle composed of a continuum of differentiated goods:

$$
C_{t}=\left[\int_{0}^{1}\left(C_{j t}\right)^{1-\frac{1}{\theta_{t}}} d j\right]^{\frac{\theta_{t}}{\theta_{t}-1}},
$$

where $C_{j t}$ is the consumption of the good produced by firm $j$. Following Steinsson (2003), Ireland (2004) and Airaudo, Nisticò, and Zanna (2006), we assume that the elasticity of substitution in demand $\left(\theta_{t}\right)$ follows a log-stationary stochastic process. This translates into a cost-push shock, which raises a non-trivial trade-off between inflation and output gap stabilization (Clarida, Gali and Gertler, 1999, Result 1). Given prices $P_{j t}$ for the $j^{\text {th }}$ good, households' demand for good $j$ and the aggregate price index $P_{t}$ read as follows:

$$
\begin{gathered}
C_{j t}=\left(\frac{P_{j t}}{P_{t}}\right)^{-\theta_{t}} C_{t}, \\
P_{t}=\left(\int_{0}^{1}\left(P_{j t}\right)^{1-\theta_{t}} d j\right)^{\frac{1}{1-\theta_{t}}} .
\end{gathered}
$$

Following Carlstrom and Fuerst (2007), we assume that households, whose labor supply is compensated at the real wage $W_{t}$, enter period $t$ with cash holdings $M_{t}, B_{t-1}$ one-period nominal bonds that pay $R_{t-1}$ gross interest $\left(1+i_{t-1}\right)$, and $A_{t-1}$ shares of stock that sell at price

\footnotetext{
${ }^{3} \mathrm{~A}$ taste shock is introduced to account for the competing effects of supply and demand side innovations on the frictionless state of the economy.
} 
$Q_{t}$ and pay dividend $D_{t}$. Before households enter the goods market, they deposit funds $M_{t}^{d}$ at financial intermediaries, which in turn remunerate them at the gross interest $R_{t}^{d}\left(=1+i_{t}^{d}\right)$. Consumption expenditures are restricted by the following liquidity constraint:

$$
P_{t} C_{t} \leq M_{t}-M_{t}^{d}+P_{t} W_{t} N_{t}
$$

The intertemporal budget constraint can be specified as:

$$
P_{t} C_{t}+P_{t} Q_{t} A_{t}+B_{t}+M_{t+1}+M_{t}^{d} \leq M_{t}+R_{t-1} B_{t-1}+P_{t} A_{t} D_{t}+R_{t}^{d} M_{t}^{d}+P_{t} Q_{t} A_{t-1}+P_{t} W_{t} N_{t}
$$

Thus, optimization conditions include the following:

$$
\begin{gathered}
\frac{N_{t}^{\eta}}{H_{t} C_{t}^{-\sigma}}=W_{t}, \\
H_{t} C_{t}^{-\sigma}=\beta E_{t}\left(\frac{R_{t} H_{t+1} C_{t+1}^{-\sigma}}{1+\pi_{t+1}}\right), \\
H_{t} C_{t}^{-\sigma}\left(Q_{t}-D_{t}\right)=\beta E_{t}\left(H_{t+1} C_{t+1}^{-\sigma} Q_{t+1}\right),
\end{gathered}
$$

where $\pi_{t}$ denotes the rate of inflation. Equilibrium in the goods market requires $Y_{t}=C_{t}$. Furthermore, equations (8) and (9) imply the usual no-arbitrage condition:

$$
Q_{t}-D_{t}=\beta E_{t}\left(\frac{1+\pi_{t+1}}{R_{t}}\right) E_{t} Q_{t+1}+\zeta_{t}
$$

where, following Smets and Wouters (2003), the term $\zeta_{t}$ accounts for the risk implied by the covariance between the stochastic discount factor and the nominal gross rate of return on stocks.

2.2. Supply Side. Following the literature on staggered pricing, we adopt the Calvo (1983) specification for the price setting mechanism. The probability that a firm optimally adjusts its price each period is $1-\omega$. A remaining fraction $\omega$ of firms does not optimally adjust, but simply updates the previous price according to an indexation rule. If a firm sets its price at time $t$, it will do so to maximize expected profits, subject to the demand function and a constant return to scale (CRS) production technology $Y_{j t}=Z_{t} N_{j t}$, where $Y_{j t}$ denotes output and $Z_{t}$ is a stochastic aggregate productivity factor. Following Ravenna and Walsh (2006), firm $j$ borrows an amount $W_{t} N_{j t}$ from intermediaries at the gross nominal interest rate $R_{t}^{l}$. It is assumed that firms are completely rationed on the equity market. If internally generated funds are not enough to finance investment, a financial gap arises that can only be filled by resorting to the credit market. Although firms could in principle issue new equity, this option is a priori ruled out, due to the possibility that new equity issues would be subject to adverse selection phenomena (see Myers and Majluf, 1984), resulting as too costly to firms. At a given share price, only overvalued firms are willing to sell their shares. As potential shareholders anticipate this fact, no trade occurs on the equity market. Under these conditions, the announcement of an equity issue is generally interpreted as bad news by investors and, in extreme situations, the stock 
market becomes a typical market for lemons. ${ }^{4}$ The cost minimization problem is specified as follows:

$$
\min R_{t}^{l} W_{t} N_{j t}+\Phi_{t}\left[Y_{j t}-Z_{t} N_{j t}\right]
$$

The real marginal cost is:

$$
\Phi_{t}=R_{t}^{l} S_{t}
$$

where $S_{t}$ equals $\frac{W_{t}}{Z_{t}}$.

2.3. The Financial Intermediary. We assume that financial intermediaries receive deposits $M_{t}^{d}$ from households and a cash injection $X_{t}\left(=M_{t+1}-M_{t}\right)$ from the monetary authority. Contextually, they supply loans $L_{t}$ to firms at the gross nominal interest rate $R_{t}^{l}$. At the end of each period, deposits $M_{t}^{d}$ together with the interest $i_{t}^{d} M_{t}^{d}$ are repaid to households. We assume that households are neither capable of monitoring the activity of entrepreneurs nor enforcing financial contracts. In this scenario, financial intermediation is required. Intermediaries operate costlessly in a competitive environment, so nominal profits in the intermediary industry are:

$$
\Pi^{i n t}=R_{t}^{l} B_{t}-R_{t}^{d} M_{t}^{d}=R_{t}^{l} P_{t} W_{t} L_{t}-R_{t}^{d} M_{t}^{d},
$$

where the following condition holds regarding resources available for lending:

$$
B_{t}=X_{t}+M_{t}^{d}
$$

Following Chowdhury, Hoffmann, and Schabert (2006), we allow for the introduction of varying degrees of interest rate changes to affect firms' lending costs. As our predecessors, rather than introducing an explicit microfoundation, we assume for simplicity that this friction can be measured by a function $\Psi_{t}$, which depends on the current risk-free interest rate, $\Psi\left(R_{t}\right)$. Log-linearization leads to:

$$
\widehat{R}_{t}^{l}=(1+\psi) \widehat{R}_{t},
$$

where $\psi\left(=\Psi_{R} R / \Psi\right)$ denotes the elasticity of the contractual interest rate to percentage changes in the policy rate. A negative value for $\psi$ indicates that a change in the risk-free interest rate is not completely passed through to the lending rate, which can be rationalized by loan price rigidities (see e.g., Hannan and Berger, 1991). Under these circumstances the cost channel is mitigated. When $\psi$ is positive, a rise in $\widehat{R}_{t}$ is even accelerated, such that the lending rate rises by more than one-to-one. This can be viewed as a reduced form relation based on financial market imperfections due to asymmetric information as accentuated in the literature on the financial accelerator (see Bernanke, Gertler and Gilchrist, 1999). This parameterization turns out to be quite convenient to assess different dynamics and policy outcomes. Moreover, empirical results support its adoption: Chowdhury, Hoffmann, and Schabert (2006) estimate an elasticity of 0.28 for Japan and 0.32 for the United States, whereas $\psi$ equals -0.45 and -0.04 in France and

\footnotetext{
${ }^{4}$ Asymmetric information is assumed to affect solely the equity market. As to the credit market, it is assumed that the banking sector has perfect information, being capable to discriminate firms on the basis of their financial structure.
} 
Germany, respectively.

2.4. Log-Linear System. The dynamic system describing the economy under scrutiny can be linearized, so that each variable is reported in terms of log-deviation from its flexibleprice equilibrium counterpart. For simplicity of exposition, in the remainder of the paper, the following notation applies:

$$
\begin{aligned}
& y_{t}=\widehat{y}_{t}-\widehat{y}_{t}^{f}, \quad q_{t}=\widehat{q}_{t}-\widehat{q}_{t}^{f}, \\
& r_{t}=\widehat{R}_{t}-\widehat{R}_{t}^{f}, \quad d_{t}=\widehat{d}_{t}-\widehat{d}_{t}^{f} .
\end{aligned}
$$

From equations (9) and (??) we derive the following relations describing the evolution of the output gap and the deviation of the stock price from its flexible-price counterpart:

$$
\begin{aligned}
& y_{t}=E_{t} y_{t+1}-\frac{1}{\sigma}\left(r_{t}-E_{t} \pi_{t+1}\right), \\
& q_{t}=(1-\beta) d_{t}+\beta E_{t} q_{t+1}-\beta\left(r_{t}-E_{t} \pi_{t+1}\right) .
\end{aligned}
$$

We assume that firms fully transfer profits in the form of dividends to the stockholders. Therefore, we impose the following aggregate resource constraint:

$$
D_{t}=Y_{t}-R_{t}^{l} W_{t} N_{t}
$$

It is straightforward to show that the following set of variables under flexible prices can be defined in terms of percentage deviation from their steady state level:

$$
\begin{aligned}
\widehat{R}_{t}^{f} & =\sigma\left(E_{t} \widehat{y}_{t+1}^{f}-\widehat{y}_{t}^{f}+g_{t}\right), \\
\widehat{y}_{t}^{f} & =\frac{1}{\sigma+\eta}\left((1+\eta) z_{t}-\widehat{R}_{t}^{f}+h_{t}\right), \\
\widehat{d}_{t}^{f} & =\widehat{y}_{t}^{f}, \\
\widehat{q}_{t}^{f} & =(1-\beta) \widehat{d}_{t}^{f}+\beta\left(E_{t} \widehat{q}_{t+1}^{f}-\widehat{R}_{t}^{f}\right),
\end{aligned}
$$

where $g_{t}=\frac{1-\rho_{h}}{\sigma} h_{t}$. Thus dividends can be transformed in terms of log-deviation from their frictionless counterpart:

$$
d_{t}=\varsigma y_{t}-\mu r_{t}
$$

where the following notation applies:

$$
\begin{aligned}
\varsigma & =1-(\theta-1)(\sigma+\eta), \\
\mu & =(\theta-1)(1+\psi) .
\end{aligned}
$$

Plugging (20) into (15) we get:

$$
q_{t}=\epsilon y_{t}-\xi r_{t}+\beta E_{t} q_{t+1}+\beta E_{t} \pi_{t+1},
$$


where:

$$
\begin{aligned}
\epsilon & =(1-\beta) \varsigma, \\
\xi & =(1-\beta) \mu+\beta .
\end{aligned}
$$

Notice that an increase in the equilibrium level of the elasticity of substitution $(\theta)$ has a twofold detrimental effect on the asset price gap: (i) via the output gap $\left(y_{t}\right)$ and (ii) via the interest rate $\left(r_{t}\right)$. The latter is substantially amplified in the presence of strong distortions in the credit market $(\psi \gg 0) .^{5}$

With regard to the supply-side block, optimization of the discounted flow of future profits under Calvo pricing and log-linearization enables us to obtain the following augmented inflationadjustment equation (see Galì and Gertler, 1999; Sbordone, 2002), which now accounts for the cost channel effect:

$$
\pi_{t}=\beta E_{t} \pi_{t+1}+\kappa(1+\psi) r_{t}+\kappa(\sigma+\eta) y_{t}+\varepsilon_{t},
$$

where $\varepsilon_{t}=\left(1-\frac{\theta}{\theta-1}\right) \frac{(1-\omega)(1-\omega \beta)}{\omega} \ln \left(\theta_{t} / \theta\right)$.

We also assume that the shocks have a stationary autoregressive representation:

$$
\left[\begin{array}{c}
\theta_{t} \\
h_{t} \\
z_{t}
\end{array}\right]=\boldsymbol{\Gamma}_{0}\left[\begin{array}{c}
\theta \\
h \\
z
\end{array}\right]+\boldsymbol{\rho}\left[\begin{array}{c}
\theta_{t-1} \\
h_{t-1} \\
z_{t-1}
\end{array}\right]+\left[\begin{array}{c}
\varrho_{t}^{\theta} \\
\varrho_{t}^{h} \\
\varrho_{t}^{z}
\end{array}\right]
$$

where

$$
\boldsymbol{\Gamma}_{0}=\left[\begin{array}{ccc}
1-\rho_{\theta} & 0 & 0 \\
0 & 1-\rho_{h} & 0 \\
0 & 0 & 1-\rho_{z}
\end{array}\right], \quad \boldsymbol{\rho}=\left[\begin{array}{ccc}
\rho_{\theta} & 0 & 0 \\
0 & \rho_{h} & 0 \\
0 & 0 & \rho_{z}
\end{array}\right], \quad\left|\rho_{i}\right|<1, \quad i=\theta, h, z
$$

and $\varrho_{t}^{i}(i=\theta, h, z)$ are iid innovations. Steady state values of other endogenous state variables are reported in Appendix A.

\section{Transmission Mechanism in a Frictionless Economy}

We turn our attention to the transmission mechanism in a frictionless economy. Under these circumstances, the interest rate consistent with the flexible-price allocation is what Woodford (2003) refers to as the Wicksellian natural rate of interest (henceforth $\widehat{R}_{t}^{f}$ ). Moreover, this interest rate is compatible with full price stability $\left(\pi_{t}=0\right)$ when we rule out the short run trade-off between inflation and output variability. ${ }^{6}$ To determine $\widehat{R}_{t}^{f}$, we consider the linear stochastic system composed of equations (16) and (17). Therefore, we can obtain the following

\footnotetext{
${ }^{5}$ Notice that, when the cost channel effect is switched off $(\psi=-1)$, the degree of competitiveness does not act as an amplifier of the transmission mechanism.

${ }^{6}$ In our setting the trade-off can be ruled out by assuming a constant elasticity of substitution between goods.
} 
solution for the natural rate of interest:

$$
\widehat{R}_{t}^{f}=\frac{\sigma(\sigma+\eta-1)\left(1-\rho_{h}\right)}{\eta+\sigma \rho_{h}} h_{t}-\frac{\sigma(1+\eta)\left(1-\rho_{z}\right)}{\eta+\sigma \rho_{z}} z_{t} .
$$

Not surprisingly, the frictionless rate of interest increases in response to a shock to the degree of impatience, while it decreases in response to a shock to productivity. Moreover, as shown in (24), technological perturbations exert a positive effect on the frictionless stock market capitalization. As to the reaction to $h_{t}$, the sign and the magnitude of the coefficient measuring the response appears to be steadily negative across all plausible parameterizations. By plugging (23) into (19) we can determine the frictionless level of stock market capitalization:

$$
\widehat{q}_{t}^{f}=\tau_{z} z_{t}-\tau_{h} h_{t}
$$

where:

$$
\begin{aligned}
\tau_{z} & =\frac{(1-\beta)(1+\eta)}{(\sigma+\eta)\left(1-\beta \rho_{z}\right)}+\frac{1+\beta(\sigma+\eta-1)}{(\sigma+\eta)\left(1-\beta \rho_{z}\right)} \frac{\sigma(1+\eta)\left(1-\rho_{z}\right)}{\left(\eta+\sigma \rho_{z}\right)}, \\
\tau_{h} & =\frac{1+\beta(\sigma+\eta-1)}{(\sigma+\eta)\left(1-\beta \rho_{h}\right)} \frac{\sigma(\sigma+\eta-1)\left(1-\rho_{h}\right)}{\left(\eta+\sigma \rho_{h}\right)}-\frac{1-\beta}{(\sigma+\eta)\left(1-\beta \rho_{h}\right)} .
\end{aligned}
$$

The effect of the taste shock on $\widehat{q}_{t}^{f}$ offers some interesting insights. In particular, two competing effects can be identified. A positive effect is channeled via the dividend process, while a negative effect comes via the no-arbitrage condition against the riskless rate of return. It turns out that when the cost channel does not matter, the overall effect is negative for $\beta$ close to 1 .

It is useful to compare the effect induced by the cost channel within a frictionless environment with respect to a situation with no supply-side effects. Equations (25) and (26) respectively report the frictionless rate of interest $\left(\widehat{R}_{t}^{f, N C C}\right)$ and the level of stock market capitalization $\left(\widehat{q}_{t}^{f, N C C}\right.$ ) under the hypothesis that the cost channel is ruled out (see also Woodford, 1999):

$$
\widehat{R}_{t}^{f, N C C}=\frac{(\sigma+\eta-1)\left(1-\rho_{h}\right) \sigma}{\sigma+\eta} h_{t}-\frac{\sigma(1+\eta)\left(1-\rho_{z}\right)}{\sigma+\eta} z_{t} .
$$

Analogously, we can determine the asset price in the absence of cost channel and cost-push shock:

$$
\widehat{q}_{t}^{f, N C C}=\frac{(1+\eta)\left(1-\beta+\beta \sigma\left(1-\rho_{z}\right)\right)}{(\sigma+\eta)\left(1-\beta \rho_{z}\right)} z_{t}-\frac{\beta(\sigma+\eta-1)\left(1-\rho_{h}\right) \sigma-1+\beta}{(\sigma+\eta)\left(1-\beta \rho_{h}\right)} h_{t} .
$$

When the cost channel is absent, the response to both sources of exogenous perturbation is lower, in absolute value. Moreover, the difference in magnitude of the reaction coefficients under the two scenarios critically depends on the degree of inertia of the shocks. The following remark formalizes the comparison between the reaction to a technological shock for both the stock price and the rate of interest. 
Remark 1. In a frictionless environment, the asset price response to a technological innovation has a greater magnitude when the cost channel matters, compared to a situation in which firms finance their production cost merely through internally generated funds. Analogous conclusions can be advanced for the frictionless rate of interest. Therefore, the following properties hold:

$$
\left.\widehat{q}_{t}^{f}\right|_{z_{t}}>\left.\left.\widehat{q}_{t}^{f, N C C}\right|_{z_{t}} \quad \widehat{R}_{t}^{f}\right|_{z_{t}}>\left.\widehat{R}_{t}^{f, N C C}\right|_{z_{t}}
$$

Along the lines traced by the literature on financial fragility (see, e.g. Kiyotaki and Moore, 1997 and Bernanke, Gertler and Gilchrist, 1999), the existence of a cost-side effect exerts a nonnegligible influence on the amplification of exogenous shocks, also in a frictionless environment. Moreover, as detailed by Woodford (1999), the more temporary the shock the higher the amplification in the face of an innovation to technology or to the degree of impatience. Nevertheless, we can point out that the effect brought by the degree of inertia of an exogenous perturbation is even stronger in the presence of the cost channel. There is no clear-cut evidence about the response of the asset price to the taste shock under the two scenarios, although for a wide range of parameterizations a greater amplification effect is detected under the cost channel.

\section{Determinacy and E-Stability Under Benchmark Interest Rate Rules}

This section is devoted to the analysis of the dynamic properties of the system summarized in equations (14), (15) and (22). To tackle the problem of REE uniqueness and E-stability (see Evans and Honkapohja, 2001), we implement two instrumental Taylor-type rules that are extensively used in the literature. As in Bullard and Mitra (2002), we first consider a contemporaneous data-based rule, then we turn our attention to the performance of a forecastbased policy function.

It is well known that determinacy is attained under the Blanchard and Kahn (1980) conditions. Let us assume the following state space form after implementing a specific interest rate rule:

$$
\boldsymbol{\Gamma} x_{t}=\boldsymbol{\Omega} E_{t} x_{t+1}+\boldsymbol{\Xi}_{\varpi_{t}}
$$

where $x_{t}=\left[\pi_{t}, y_{t}, q_{t}\right]^{\prime}$ and $\varpi_{t}$ is a vector of shocks. In our case, in the absence of any inertial effect in the model economy and in the policy reaction functions under scrutiny, REE uniqueness is simply achieved if the matrix $\Gamma^{-1} \boldsymbol{\Omega}$ has real parts of eigenvalues lying inside the unit circle. It is important to recall that we deal with a $3 \times 3$ system. Therefore, a third degree characteristic polynomial, denoted by $\digamma(\lambda)$, is retrievable from the matrix under scrutiny, where $\lambda$ represents a generic eigenvalue. Under these circumstances, a necessary condition for determinacy is:

$$
\operatorname{sign}[\digamma(-1)]=\operatorname{sign}[\digamma(1)] .
$$

For condition (27) to be also sufficient, we need the inflection point of the curve associated with the polynomial to lie within the interval $[-1,1]$. 
As to E-stability, the minimum state variable (MSV) solution takes the following form:

$$
x_{t}=\Pi \varpi_{t} .
$$

Agents are assumed to form expectations by relying on the perceived law of motion (PLM), $E_{t} x_{t+1}=\widehat{\Upsilon}+\widehat{\Pi} \rho \varpi_{t}$. Consequently, the actual law of motion (ALM) reads as follows:

$$
x_{t}=\Gamma^{-1} \boldsymbol{\Omega}\left(\Upsilon+\Pi \boldsymbol{\rho} \varpi_{t}\right)+\Gamma^{-1} \Xi_{\varpi_{t}}
$$

The T-mapping from the PLM to the ALM is:

$$
\begin{aligned}
& T(\widehat{\mathbf{\Pi}})=\boldsymbol{\Gamma}^{-1}(\boldsymbol{\Omega} \boldsymbol{\Pi} \boldsymbol{\rho}+\boldsymbol{\Xi}) \\
& T(\widehat{\boldsymbol{\Upsilon}})=\boldsymbol{\Gamma}^{-1} \boldsymbol{\Omega} \Upsilon
\end{aligned}
$$

The MSV-REE is:

$$
\begin{aligned}
\boldsymbol{\Gamma}^{-1}(\boldsymbol{\Omega} \boldsymbol{\Pi} \boldsymbol{\rho}+\boldsymbol{\Xi}) & =\boldsymbol{\Pi}, \\
\boldsymbol{\Gamma}^{-1} \boldsymbol{\Omega} \Upsilon & =0 .
\end{aligned}
$$

According to Evans and Honkapohja (2001), the MSV-REE is E-stable when the following matrix, evaluated at the REE, has eigenvalues with real parts less than 1 :

$$
\begin{aligned}
& D T_{\Pi}(\boldsymbol{\Pi})=\boldsymbol{\rho}^{\prime} \otimes \boldsymbol{\Gamma}^{-1} \boldsymbol{\Omega} \\
& D T_{\boldsymbol{\Upsilon}}(\mathbf{\Upsilon})=\boldsymbol{\Gamma}^{-1} \boldsymbol{\Omega} .
\end{aligned}
$$

Since $\boldsymbol{\rho}^{\prime}$ has all roots with real parts less than 1, a necessary and sufficient condition for Estability of the MSV-REE is that $\mathbf{J}\left(=\boldsymbol{\Gamma}^{-1} \boldsymbol{\Omega}-\mathbf{I}\right)$ has all roots with negative real parts. From the Routh Theorem (see Gandolfo, 1996) all roots of $\mathbf{J}$ have negative real parts if and only if the following three conditions hold:

$$
\begin{aligned}
\operatorname{Det}(\mathbf{J}) & <0 \\
\operatorname{Tr}(\mathbf{J}) & <0 \\
S_{2}(\mathbf{J}) \operatorname{Tr}(\mathbf{J})-\operatorname{Det}(\mathbf{J}) & <0
\end{aligned}
$$

where $S_{2}(\mathbf{J})$ is the sum of the $2 \times 2$ principal minors of $\mathbf{J}$.

In the remainder of the paper, we study determinacy and E-stability conditions under two benchmark policy reaction functions. Given the dimension of our system, the analysis is partly based on the evidence retrievable from simulation exercises. The calibrated values for $\sigma$ and $\kappa$ turn out to be crucial for pursuing a numerical exploration. In the remainder of the paper we alternatively consider the parameterizations suggested by Woodford (1999) (W), Clarida, Gali, and Gertler (1999) (CGG), and McCallum and Nelson (1999) (MN), summarized in Table 1. Moreover we assume that $\beta=0.99, \eta=2$ and $\theta=3$, if not stated otherwise. 


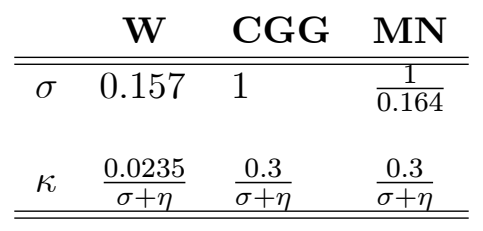

Table 1: Calibrations

4.1. Contemporaneous Data Rule. We first consider a Taylor rule that reacts to contemporaneous inflation, output gap and asset prices misalignments:

$$
r_{t}=\chi_{\pi} \pi_{t}+\chi_{y} y_{t}+\chi_{q} q_{t}
$$

To analyze determinacy and stability under learning, we write the system in state space form:

$$
\begin{aligned}
\boldsymbol{\Gamma}_{c} x_{t} & =\boldsymbol{\Omega}_{c} E_{t} x_{t+1}+\boldsymbol{\Xi}_{c} u_{t}, \\
u_{t} & =\boldsymbol{\Xi}_{\rho} u_{t-1}+e_{t}, \quad e_{t} \stackrel{i i d}{\sim} N\left(0, \sigma_{e}^{2}\right),
\end{aligned}
$$

where $x_{t}=\left[\pi_{t}, y_{t}, q_{t}\right]$ and:

$$
\begin{aligned}
& \boldsymbol{\Gamma}_{c}=\left[\begin{array}{ccc}
1-\kappa(1+\psi) \chi_{\pi} & -\kappa\left(\sigma+\eta+(1+\psi) \chi_{y}\right) & -\kappa(1+\psi) \chi_{q} \\
\frac{\chi_{\pi}}{\sigma} & \left(1+\frac{\chi_{y}}{\sigma}\right) & \frac{\chi_{q}}{\sigma} \\
\xi \chi_{\pi} & \xi \chi_{y}-\epsilon & \left(1+\xi \chi_{q}\right)
\end{array}\right], \\
& \boldsymbol{\Omega}_{c}=\left[\begin{array}{ccc}
\beta & 0 & 0 \\
\frac{1}{\sigma} & 1 & 0 \\
\beta & 0 & \beta
\end{array}\right], \quad \boldsymbol{\Xi}_{c}=\mathbf{I}_{3 \times 3}, \quad \boldsymbol{\Xi}_{\rho}=\rho \mathbf{I}_{3 \times 3} .
\end{aligned}
$$

To ensure equilibrium uniqueness and stability under adaptive learning, we need to verify that the conditions reported are fulfilled. Retrieving analytical conditions for a $3 \times 3$ system loses much of the usual appeal in terms of the power to draw clear conclusions. We find more intuitive to plot the regions of determinacy and E-stability through a numerical simulation of the model over a wide parameter sub-space for the policy rule's coefficients.

Nonetheless, we find that some appealing analytical results can be provided by considering a rule responding only to the rate of inflation. The resulting conditions retain considerable importance for those monetary authorities exclusively or primarily concerned with inflation stabilization. Brückner and Schabert (2003) and Llosa and Tuesta (2007) show that the cost channel modifies the standard conditions for determinacy and learnability when the central bank operates with either instrument or target rules. Their analysis is based on a NK model featuring a cost channel à la Ravenna and Walsh (2006). This is to say that the degree of passthrough $\psi$ from policy to bank lending rates can either take value -1 (no cost channel) or 0 (cost channel with perfect pass-through). The following proposition shows that traditional conditions for determinacy and E-stability are further affected when the whole spectrum of values for 
the pass-through parameter is considered. It turns out that, when the central bank sets the policy instrument in response to the rate of inflation, the width of the region of determinacy dramatically depends on the level of pass-through. Under these circumstances, it is extremely important to assess the magnitude of the cost channel effect.

Proposition 2. Under a contemporaneous data interest rate rule responding to the rate of inflation, the following conditions ensuring equilibrium uniqueness can be identified:

- Iff $\psi>\frac{\eta}{\sigma}$ :

$$
\chi_{\pi}<\widehat{\chi}_{\pi}=\frac{\sigma(\beta-1)}{\kappa(\eta-\sigma \psi)}
$$

- Iff $\psi>\frac{\eta-\sigma}{2 \sigma}$ :

$$
\chi_{\pi}<\widetilde{\chi}_{\pi}=\frac{2 \sigma(1+\beta)+\kappa(\sigma+\eta)}{\kappa(\sigma(1+2 \psi)-\eta)} .
$$

- Iff $\psi<\frac{\eta-\sigma}{2 \sigma}$ :

$$
\chi_{\pi}>\bar{\chi}_{\pi}=1
$$

Proof. See Appendix A.

To attain stability under adaptive learning, we need to check that the eigenvalues of $\mathbf{J}_{c 33}-\mathbf{I}$ are negative. It can be easily shown that the sufficient and necessary conditions require both $C_{c}>0$ and $D_{c}>0$ in the characteristic polynomial retrievable from $\mathbf{J}_{c 33}-\mathbf{I}$ :

$$
\lambda^{2}+C_{c} \lambda+D_{c}=0
$$

where

$$
\begin{aligned}
C_{c} & =\frac{\sigma(1-\beta)+\kappa \chi_{\pi}(\eta-\sigma \psi)+\kappa(\eta+\sigma)\left(\chi_{\pi}-1\right)}{\sigma+\kappa \chi_{\pi}(\eta-\sigma \psi)}, \\
D_{c} & =\frac{\kappa(\sigma+\eta)\left(\chi_{\pi}-1\right)}{\sigma+\kappa \chi_{\pi}(\eta-\sigma \psi)} .
\end{aligned}
$$

Conditions for E-stability are fully nested in those for determinacy. In Appendix A we report a proposition and a corollary regarding the E-stability conditions under a contemporaneous interest rate rule. Moreover, the following corollary provides a series of response intervals to contemporaneous inflation that ensure equilibrium uniqueness, by taking into consideration the degree of pass-through from the official rate to the credit market interest rate.

Corollary 3. The set of conditions stated in the proposition above allows us to determine an interval for critical values of the pass-through:

1. Iff $\psi<\frac{\eta-\sigma}{2 \sigma}$ the system will always be determinate if $\chi_{\pi}>1$.

2. Iff $\frac{\eta-\sigma}{2 \sigma} \leq \psi<\frac{\sigma(1-\beta)+\left(\kappa \eta \sigma^{-1}+\kappa+3 \beta+1\right) \eta}{\kappa \sigma+4 \sigma \beta+\kappa \eta}$ the response coefficient to inflation has to lie within the area between the locus $\tilde{\chi}_{\pi}$ and the bottom limit represented by $\bar{\chi}_{\pi}=1$; 
3. Iff $\frac{\sigma(1-\beta)+\left(\kappa \eta \sigma^{-1}+\kappa+3 \beta+1\right) \eta}{\kappa \sigma+4 \sigma \beta+\kappa \eta} \leq \psi<\frac{\eta}{\sigma}+\frac{(1-\beta)}{\kappa}$ the response coefficient to inflation has to lie within the area between the locus $\widehat{\chi}_{\pi}$ and the bottom limit represented by $\bar{\chi}_{\pi}=1$;

4. Iff $\psi \geq \frac{\eta}{\sigma}+\frac{(1-\beta)}{\kappa}$ determinacy is never attained.

\section{Proof. See Appendix A.}

Conditions reported in Corollary 3 underline the importance of assessing the degree of passthrough when setting up the response coefficient to contemporaneous inflation. We perform a series of numerical exercises to explore the conditions for determinacy and E-stability under the Taylor rule expressed in (28). Therefore, we consider a monetary authority that not only responds to the rate of inflation, but also to output gap and asset prices misalignments. We rely on the set of calibrated parameters proposed by McCallum and Nelson (1999) and Woodford (1999). Results from the MN parameterization are reported in the main text. ${ }^{7}$ We perform each numerical exercise under three different values of the pass-through parameter $(\psi=\{-1,0,0.5\})$. Thus, we can readily observe the effect induced by an increasing importance of the cost channel effect. In line with Bullard and Mitra (2002), the autoregressive coefficients of the structural shocks are set to produce log-stationary perturbations.

Moreover, the red-pink (darker) area denotes the space of indeterminacy and E-instability, whereas the turquoise area (lighter) is associated with the space of indeterminacy and E-stability under learning. The white area denotes combinations of responses that ensure both determinacy and E-stability. The figures in each panel will be referred to as (a), (b) and (c) from left to right.
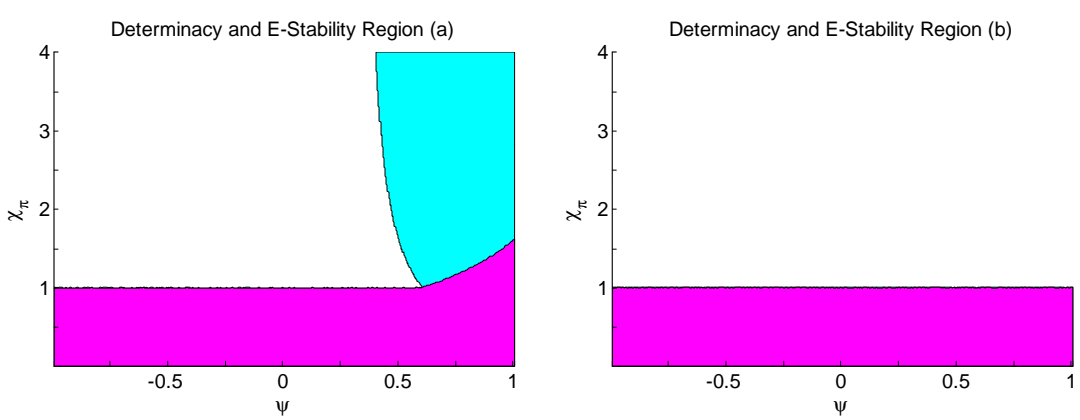

Figure 1: Regions of determinacy and E-stability [MN (a) and W (b) calibrations]

Figure 1 plots E-stability and determinacy regions for different values of the pass-through when the central bank responds only to the inflation rate. On the one hand, determinacy cannot be achieved when $\psi>\frac{\eta}{\sigma}+\frac{(1-\beta)}{\kappa}$ (about 0.60 under MN calibration). On the other hand, determinacy will always be achieved if $\psi<\frac{\eta-\sigma}{2 \sigma}$ (about -0.34 under MN calibration). Between these two thresholds there are two conditions that prevent the central bank from responding too strongly to inflation. As the value of the pass-through is always smaller than $\frac{\eta}{\sigma}$ under Woodford

\footnotetext{
${ }^{7}$ The calibration proposed in McCallum and Nelson (1999) is often implemented in adaptive learning studies, especially when focusing on the efficiency of different Taylor-type rules in ensuring REE uniqueness and learnability. Numerical simulations for Woodford (1999) calibration are available from authors upon request.
} 
(1999) calibration, the standard condition for determinacy, formalized in the Taylor principle, is never altered when the central bank responds only to inflation. To ensure determinacy, when the value of pass-through is greater than $\frac{\eta}{\sigma}+\frac{(1-\beta)}{\kappa}$, the central bank has to respond to either the output gap or asset prices, along with responding to the rate of inflation.
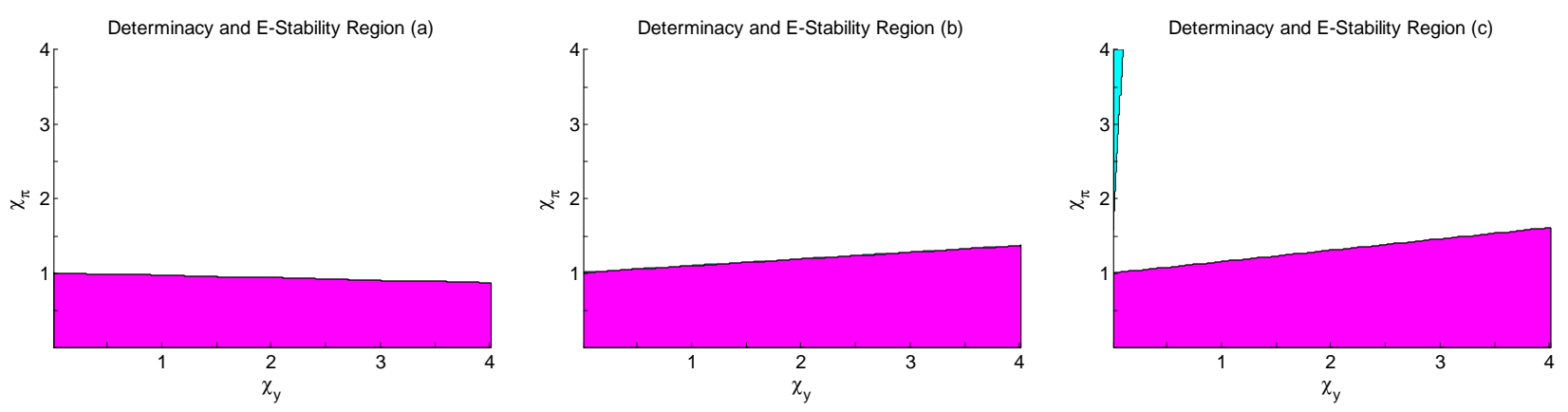

Figure 2: Regions of determinacy and E-stability with $\chi_{q}=0$

In the second round of simulations we determine conditions for determinacy and E-stability in the $\left\{\chi_{\pi}, \chi_{y}\right\}$ space. In the first exercise (Figure 2) we set $\chi_{q}=0$, whereas in the second exercise (Figure 3 ) we impose $\chi_{q}=0.5$. Figure 2(a) plots the combinations of $\left\{\chi_{\pi}, \chi_{y}\right\}$ ensuring determinacy and E-stability when the cost channel is ruled out $(\psi=-1)$. The resulting conditions can be summarized in the well known Taylor principle. Figures $2 \mathrm{a}$ and $2 \mathrm{~b}$ show that, as the degree of pass-through increases, the Taylor principle is no longer sufficient to guarantee determinacy. Turning the attention to the combinations ensuring equilibrium uniqueness in the presence of a cost channel effect with perfect pass-through, it is evident how the area of indeterminacy enlarges, as it shifts up on the $\chi_{\pi}$ axis and decreases in $\chi_{y}$. Given that the nominal rate of interest has a direct effect on the rate of inflation via bank lending, a higher degree of reactiveness is required to avoid non-fundamental fluctuations in output and inflation. For a higher degree of pass-through $(\psi=0.5)$, which reflects strong credit market distortions, we can better appreciate the intuition behind Corollary 3. In this case, responding only to inflation might not ensure equilibrium uniqueness. In fact, Figure 2(c) shows that a region of indeterminacy arises along the $\chi_{\pi}$ axis, whenever the central bank does not react to the output gap or when it reacts too weakly $\left(\chi_{y} \approx 0\right)$. A monetary contraction designed to fight inflationary pressures results in even higher inflation due to the fact that interest rate has a direct effect on inflation in the NKPC. When there is a strong pass-through, the price puzzle effect is even more accentuated. Reacting to the contemporaneous rate of inflation will lead to higher inflation in the next period, eventually driving the system in the area of indeterminacy.

We can observe that in most of the cases the area of E-stability is matches the one of indeterminacy. The possibility of learnable sunspot equlibria can occur only when the degree of pass-through is very high [as in Figure 2(c)]. The spike of indeterminacy occurring for a low response to asset prices misalignments and output gap is E-stable. ${ }^{8}$

\footnotetext{
${ }^{8}$ If we would assume the PLM of the MSV-REE form (no constant in the PLM) then results for E-stability would change. For $\rho<0.98$ the area denoting E-unstable equilibria would shrink when $\chi_{q}$ and $\chi_{y}$ increase. However, it would enlarge as the degree of distortion $\psi$ increases. This result is robust across different calibrations.
} 

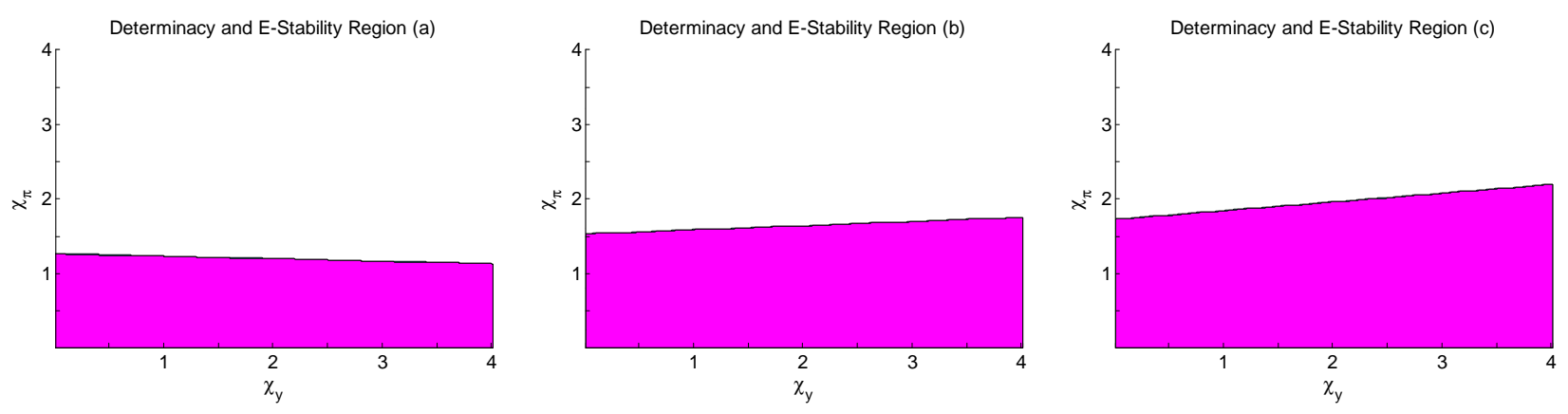

Figure 3: Regions of determinacy and E-stability with $\chi_{q}=0.5$

In Figure 3, we assume that the monetary authority implements a rule featuring a positive response to asset prices misalignments $\left(\chi_{q}=0.5\right)$. Overall, responding to asset prices enlarges the area of indeterminacy. In particular, this shifts up and its slope increases in $\psi$. Nevertheless, with a positive $\chi_{q}$ the central bank excludes the likelihood of equilibrium multiplicity in correspondence with a small response to the output gap, along the $\chi_{\pi}$ axis. This is particularly evident from the comparison of Figures 2(c) and 3(c).
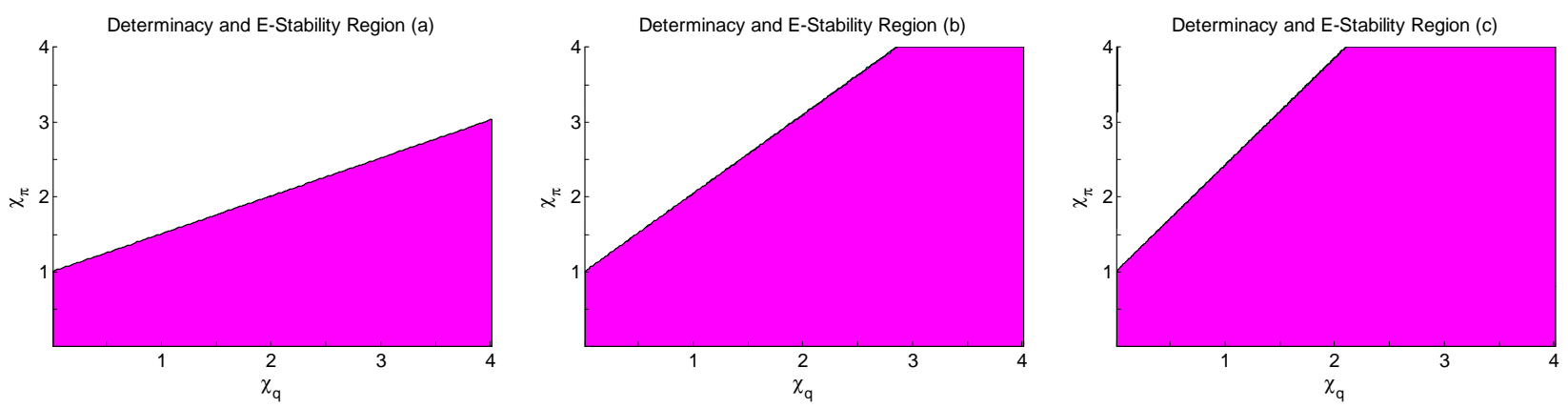

Figure 4: Regions of determinacy and E-stability with $\chi_{y}=0$
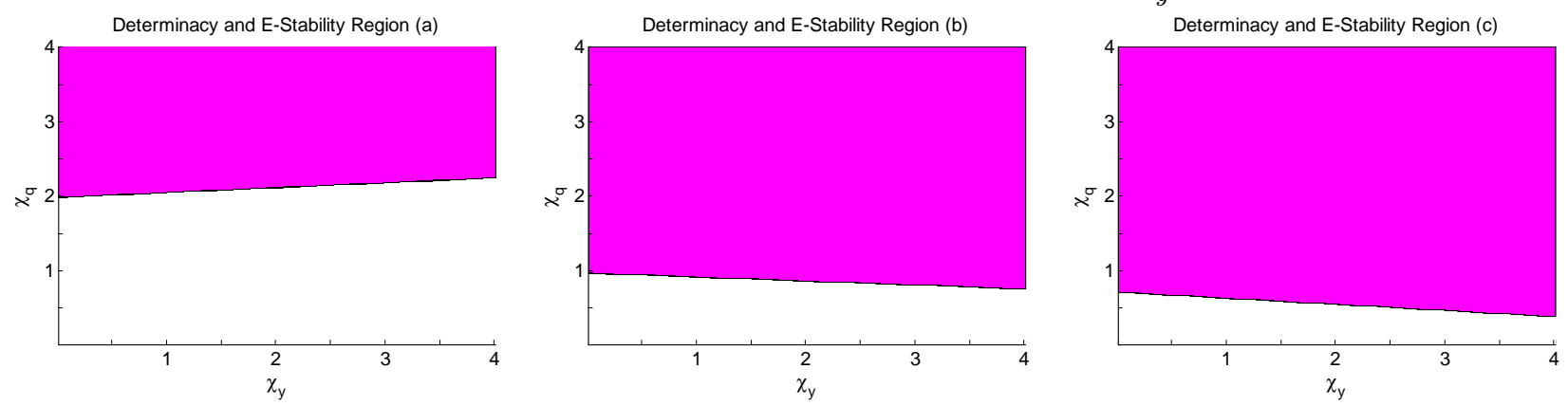

Figure 5: Regions of Determinacy and E-Stability with $\chi_{\pi}=2$

Figure 5 suggests that the central bank should never react too strongly to asset prices misalignments. The maximum threshold ensuring both determinacy and E-stability is always higher for $\chi_{y}$ rather than $\chi_{q}$. Nevertheless, if we repeat the same exercise under a higher $\chi_{\pi}$ [e.g. $\chi_{\pi}=3$ in Figure 5(c)] an area of indeterminacy will be detectable in correspondence of low values of both coefficients. In this case, we would observe that an approximately four times 
lower $\chi_{q}$ is required to drive the system into the area of determinacy, compared to the necessary $\chi_{y}$. This is even more evident under a forward looking rule.

4.2. Forward Looking Expectational Rule. Next, we consider a forward looking rule where the monetary authority reacts to future expected inflation, output gap and asset prices misalignments:

$$
r_{t}=\chi_{\pi} E_{t} \pi_{t+1}+\chi_{y} E_{t} y_{t+1}+\chi_{q} E_{t} q_{t+1} .
$$

The model in state space form can be written as:

$$
\begin{aligned}
\boldsymbol{\Gamma}_{f} x_{t} & =\boldsymbol{\Omega}_{f} E_{t} x_{t+1}+\boldsymbol{\Xi}_{f} u_{t}, \\
u_{t} & =\boldsymbol{\Xi}_{\rho} u_{t-1}+e_{t}, \quad e_{t} \stackrel{i i d}{\sim} N\left(0, \sigma_{e}^{2}\right),
\end{aligned}
$$

where $x_{t}=\left[\pi_{t}, y_{t}, q_{t}\right]$ and

$$
\begin{aligned}
& \boldsymbol{\Omega}_{f}=\left[\begin{array}{ccc}
\beta+\kappa(1+\psi) \chi_{\pi} & \kappa(1+\psi) \chi_{y} & \kappa(1+\psi) \chi_{q} \\
\frac{1}{\sigma}\left(1-\chi_{\pi}\right) & 1-\frac{\chi_{y}}{\sigma} & -\frac{\chi_{q}}{\sigma} \\
\beta-\xi \chi_{\pi} & -\xi \chi_{y} & \beta-\xi \chi_{q}
\end{array}\right], \\
& \boldsymbol{\Gamma}_{f}=\left[\begin{array}{ccc}
1 & -\kappa(\sigma+\eta) & 0 \\
0 & 1 & 0 \\
0 & -\epsilon & 1
\end{array}\right], \quad \boldsymbol{\Xi}_{f}=\mathbf{I}_{3 \times 3}, \quad \boldsymbol{\Xi}_{\rho}=\rho \mathbf{I}_{3 \times 3} .
\end{aligned}
$$

Again, to assess the effects brought by the cost channel in terms of REE uniqueness and learnability, we find intuitive to study the system under a rule responding only to expected inflation. The following proposition shows that traditional conditions for determinacy and Estability are further altered when different degrees of distortion affect the credit market.

Proposition 4. Under an interest rate rule responding only to expected inflation in the presence of a cost channel, the necessary and sufficient conditions guaranteeing determinacy can be stated as follows:

- Iff $\psi>-1$ :

$$
\begin{aligned}
& \chi_{\pi}=\bar{\chi}_{\pi}>1 \\
& \chi_{\pi}<\widehat{\chi}_{\pi}=\frac{1-\beta}{\kappa(\psi+1)}
\end{aligned}
$$

- Iff $\psi<\frac{\eta-\sigma}{2 \sigma}$ :

$$
\chi_{\pi}<\tilde{\chi}_{\pi}=\frac{2 \sigma(1+\beta)+\kappa(\sigma+\eta)}{\kappa(\sigma+\eta)-2 \kappa \sigma(1+\psi)}
$$

\section{Proof. See Appendix A.}

It can be shown that the conditions ensuring E-stability are fully encompassed by those guaranteeing equilibrium uniqueness. In Appendix A we report a proposition and a corollary 
regarding the E-stability conditions under a forward looking interest rate rule. Moreover, the following corollary identifies the range of responses to expected inflation ensuring equilibrium uniqueness and E-stability, depending on the degree of pass-through from the official rate to the credit market interest rate.

Corollary 5. The set of conditions stated in the proposition above allows us to determine an interval for critical values of the pass-through:

1. Iff $-1<\psi \leq \frac{(1-\beta)(\sigma+\eta)}{4 \sigma+\kappa(\sigma+\eta)}-1$ the response coefficient to inflation has to lie within the area between the locus $\widetilde{\chi}_{\pi}$ and the bottom limit represented by $\bar{\chi}_{\pi}=1$.

2. Iff $\frac{(1-\beta)(\sigma+\eta)}{4 \sigma+\kappa(\sigma+\eta)}-1<\psi<\frac{1-\beta-\kappa}{\kappa}$ the response coefficient has to be lie within the area between the locus $\hat{\chi}_{\pi}$ and the bottom limit represented by $\bar{\chi}_{\pi}=1$.

3. Iff $\psi \geq \frac{1-\beta-\kappa}{\kappa}$ determinacy is never attained.

Proof. See Appendix A.
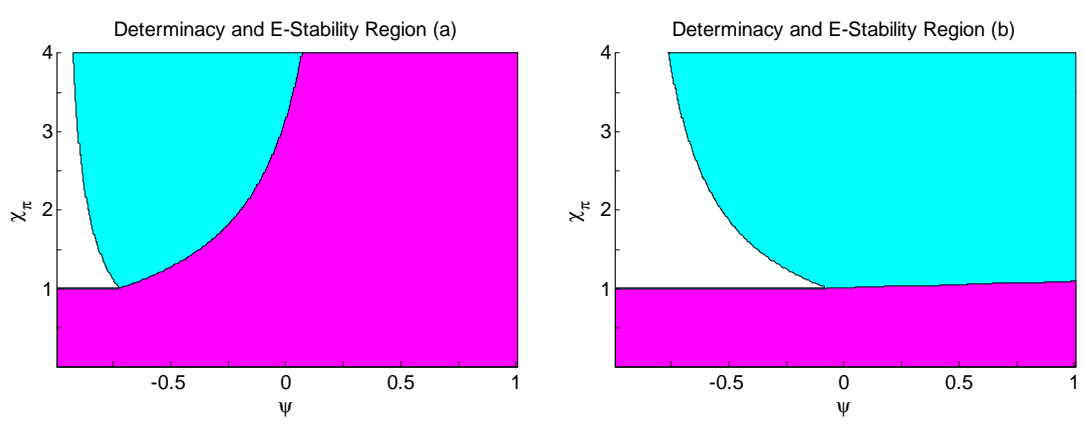

Figure 6: Regions of determinacy and E-stability [MN (a) and W (b) Calibrations]

Conditions reported in Corollary 5 point out the importance of considering the degree of pass-through when setting up the response coefficient to the expected future inflation. Figure 6 shows the dynamic properties of the system in the $\left\{\psi, \chi_{\pi}\right\}$ space. If the central bank reacts only to the rate of inflation, a unique REE can be obtained only for low values of the pass-through. As detailed in Corollary 5, the monetary authority has to avoid a strong reaction to inflation as this might shift the system into the area of indeterminacy. ${ }^{9}$

Figures 7(b) and 7(c) show that the system delivers an indeterminate outcome in the presence of the cost channel, over the whole spectrum of $\chi_{\pi}$, when the central bank does not respond either to expected assets prices misalignments or to output gap. Again, following a rule that responds only to expected inflation the central bank will trigger inflationary pressures via the banking system. This is especially evident when pricing behavior in this sector amplifies movements in the policy rate. In addition, the spike of indeterminacy rising along the $\chi_{\pi}$-axis is greater than the one obtained under a contemporaneous rule. It is also important to stress that to rule out indeterminacy the response coefficient to the expected output gap has to increase in the degree of pass-through.

\footnotetext{
${ }^{9}$ It is also worth pointing out that the determinacy area is wider under the set of calibrated parameters suggested by Woodford (1999).
} 

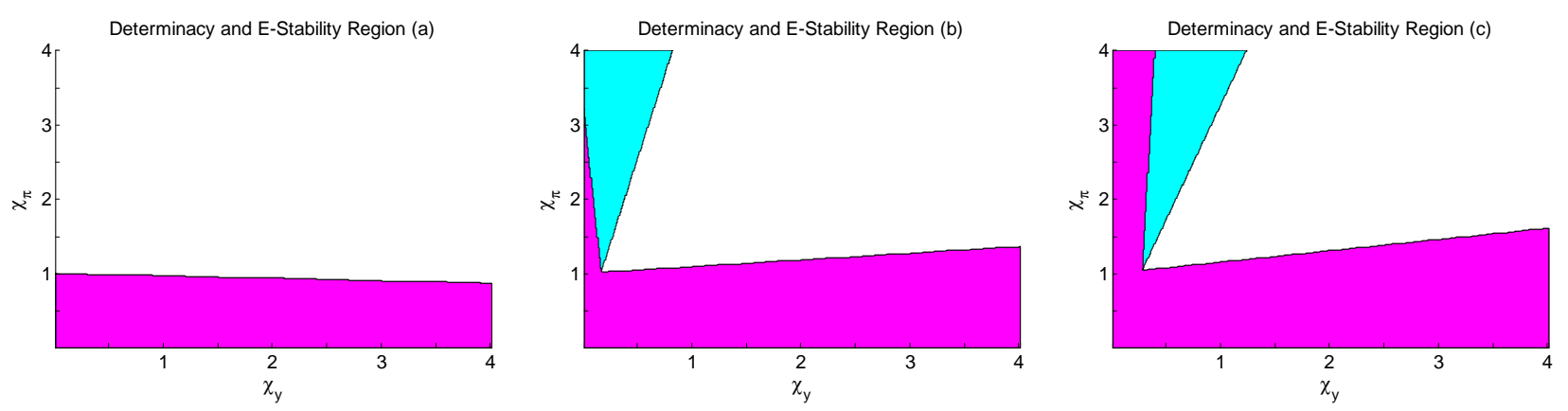

Figure 7: Regions of determinacy and E-stability with $\chi_{q}=0$

Simulations reported in Figure 7 also confirm the basic results observed in Figure 6. Under large distortions $(\psi>0)$, as those reflected in Figure $7(\mathrm{c})$, the central bank has to respond quite strongly to the output gap and to make sure that its reaction to inflation is not too strong, as this would lead to an indeterminate outcome. It is interesting to notice that significant part of the spike of indeterminacy for low responses to asset prices misalignments and output gap is E-stable.
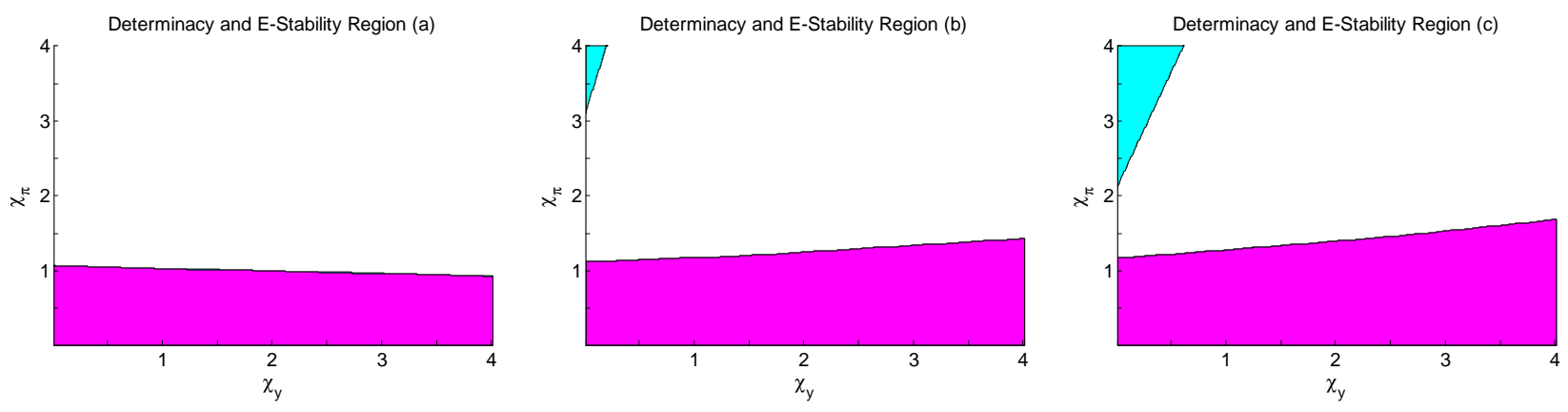

Figure 8: Regions of determinacy and E-stability with $\chi_{q}=0.1$

If we replicate the last exercise by setting $\chi_{q}=0.1$ (Figure 8), the indeterminacy area previously detectable for low values of $\chi_{y}$ is now significantly smaller. Relatively low values of the reaction coefficient attached to expected asset prices misalignments allow the central bank not to respond to the output gap. At the same time, the response to inflation should be constrained within a certain range. In this case, there is a higher probability to attain a unique REE if the central bank responds to asset prices misalignments. Moreover, by responding to asset prices the E-instability area previously detectable for low values of $\chi_{y}$ significantly decreases or is compleately removed (see also Figure 10). 

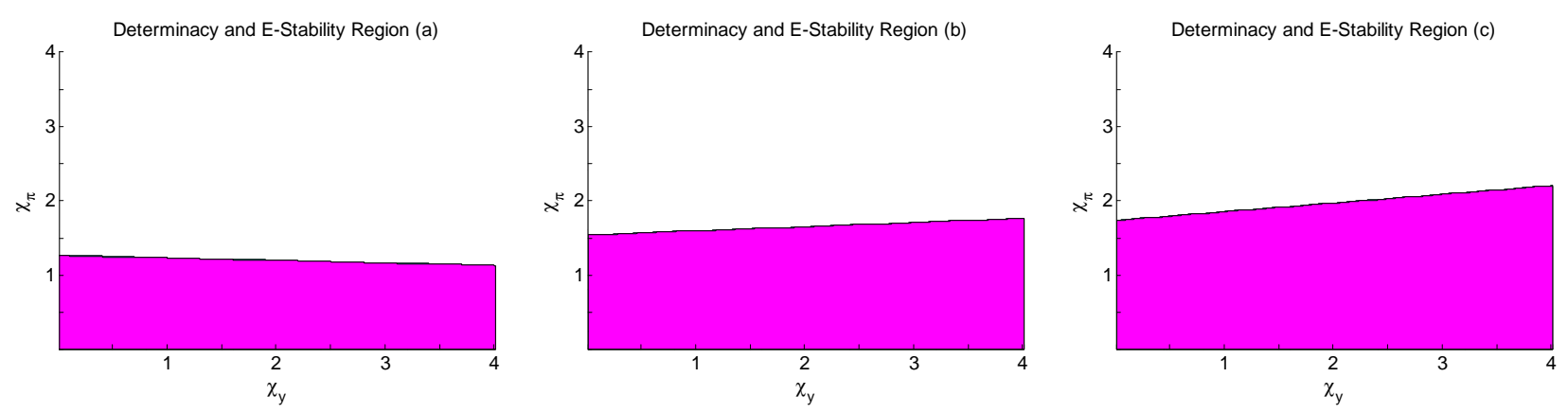

Figure 9: Regions of determinacy and E-stability with $\chi_{q}=0.5$

As shown in Figure 9, a greater response to asset price misalignments $\left(\chi_{q}=0.5\right)$ would almost completely remove the area of instability arising at low values of $\chi_{y}$. Nonetheless, the minimum response to inflation has to be stronger, compared to the previous exercise.

To confirm the results from previous simulations, we also report determinacy conditions in the $\left\{\chi_{q}, \chi_{\pi}\right\}$ space for $\chi_{y}=0$ (Figure 10), while Figure 11 reports the area of determinacy in the $\left\{\chi_{q}, \chi_{y}\right\}$ space for $\chi_{\pi}=2$. Figure 10 shows that a higher response to asset prices has to be accompanied by a higher response to inflation to ensure equilibrium uniqueness. Moreover, Figure 11 shows that the area of determinacy significantly narrows down as the degree of passthrough increases and reflects a highly distortive allocation mechanism in the credit market. Ceteris paribus $\left(\chi_{\pi}=2\right.$ and $\left.\psi=0.5\right)$, the value of $\chi_{q}$ required to shift the system into the area of determinacy is twice as small as the necessary $\chi_{y}$, although the range of responses ensuring determinacy is higher in the second case.
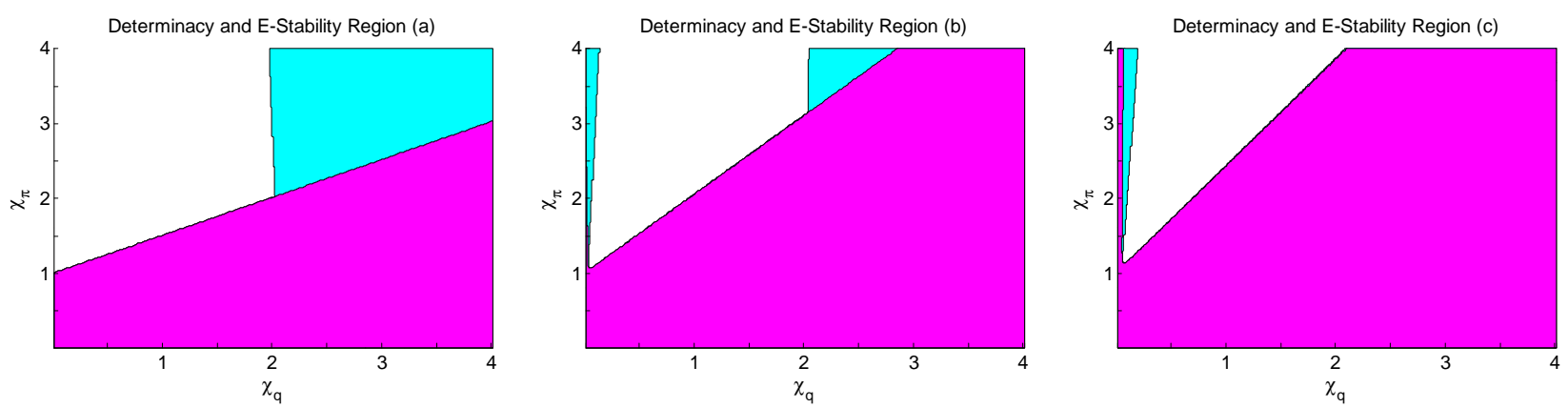

Figure 10: Regions of determinacy and E-stability with $\chi_{y}=0$
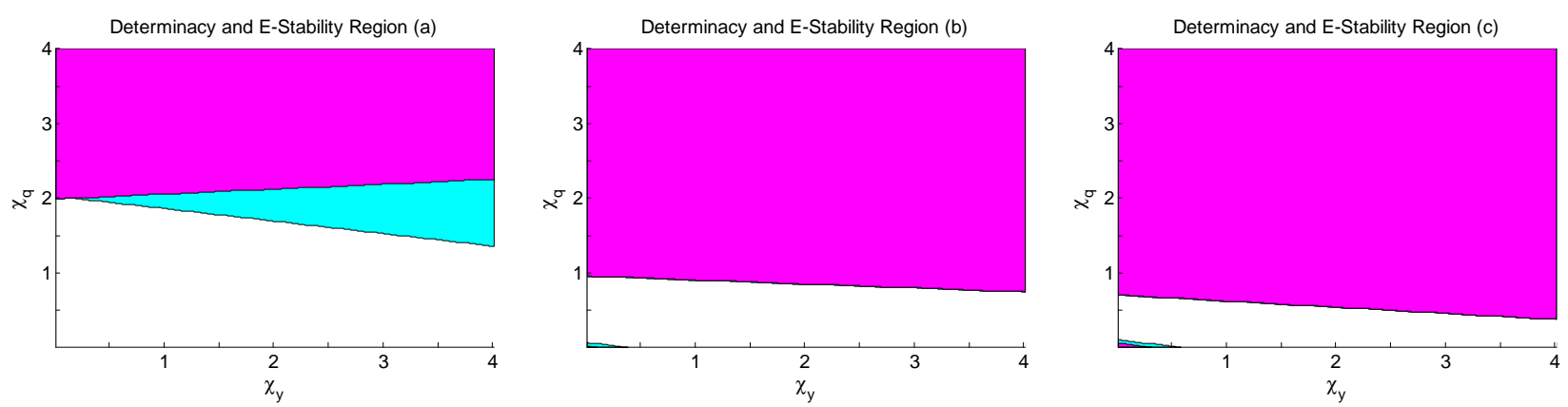

Figure 11: Regions of determinacy and E-stability with $\chi_{\pi}=2$ 
The analysis of the conditions for equilibrium uniqueness offers an interesting picture. It turns out that even a low response to asset prices misalignments helps at ruling out the area of indeterminacy that arises if the monetary authority reacts only to the rate of inflation. Overall, a joint response to both $E_{t} q_{t+1}$ and $E_{t} y_{t+1}$ generally leads to a wider area of determinacy. The central bank achieves greater benefits from the implementation of a rule featuring a relatively lower response to the expected output gap. Otherwise, if the monetary authority does not respond to asset prices misalignments, then it should attach a significantly stronger response to $E_{t} y_{t+1}$. It is interesting to point out that, under the Woodford (1999) calibration (Appendix $\mathrm{B}$ ), the area of determinacy is much wider when the central bank responds to asset prices rather than to the output gap. Simulations under this set of parameters confirm the importance of reacting to either asset prices or output gap in the presence of strong credit market distortions.

4.3. Discussion. As our analytical and numerical results show, standard conditions for determinacy and E-stability are modified when the cost channel matters. We show that responding to asset prices misalignments in the presence of strong distortions affecting the credit market allows the central bank to restore REE uniqueness and E-stability. This is particularly evident when the monetary authority implements a forward looking rule (32), as the system is more sensitive to changes in the degree of pass-through. In this case, even a weak cost channel effect introduces significant changes in the conditions for determinacy and E-stability. In fact, under a contemporaneous data rule, a higher $\psi$ is necessary to observe a spike of indeterminacy for low values of the response coefficient attached to the output gap. This is to say that, especially under uncertainty regarding the magnitude of the cost channel effect, a monetary authority is better off by responding to current rather than to forecast data. This strategy generally allows the central bank to face a wider area of determinacy.

In standard NK frameworks with no capital market imperfections, dividends are negatively related to the output gap. As marginal costs are proportional to the output gap, an interest rate rule that responds positively to (expected or current) stock price deviations from their frictionless level is a rule that responds positively to firms' profitability. This amounts to say that the central bank responds negatively to the underlying distortion-the marginal cost. This is exactly the opposite of what should be done to achieve stabilization. When a cost channel effect is at work, this mechanism no longer holds. This is especially evident for high values of the pass-through, which reflect a distortive allocation mechanism in the credit market. In this case, the monetary instrument affects positively marginal costs, hence a monetary innovation exerts a direct influence on inflation dynamics in the same direction. At the same time, inflationary pressures are scaled down if the central bank responds positively to asset prices, as this reflects a negative response to the output gap that balances the cost channel effect in the NK curve. In presence of cost-side effects, reacting to either contemporaneous or expected asset prices misalignments shifts upwards the set of conditions that bind the response to the rate of inflation from above. This mechanism is at the root of the gain in determinacy when we consider a positive response to current or expected asset prices misalignments. Therefore, if the cost channel matters, we draw opposite policy prescriptions with respect to those advanced by Carlstrom 
and Fuerst (2007) in their baseline framework. As a matter of fact, they assert that one shortcoming of the benchmark model is that in response to a monetary contraction, marginal cost falls so sharply that profits actually rise. ${ }^{10}$ Our model abstracts from this criticism, as profitability is directly affected by the nominal interest rate via a cost channel effect.

\section{Welfare Analysis}

We shift our focus on the welfare implications of the two benchmark rules under scrutiny. In line with Orphanides and Williams (2007), we analyze the loss incurred by the monetary authority under different stabilization objectives. The following loss specifications are considered:

$$
\begin{aligned}
& L_{1}=\pi_{t}^{2}+\varphi^{y} y_{t}^{2}, \\
& L_{2}=\pi_{t}^{2}+\varphi^{y} y_{t}^{2}+\varphi^{r}\left(\Delta r_{t}\right)^{2}, \\
& L_{3}=\pi_{t}^{2}+\varphi^{y} y_{t}^{2}+\varphi^{q} q_{t}^{2}+\varphi^{r}\left(\Delta r_{t}\right)^{2}, \\
& L_{4}=\pi_{t}^{2}+\varphi^{y} y_{t}^{2}+\varphi^{q} q_{t}^{2} .
\end{aligned}
$$

Calibration of the coefficients in the loss function is still an open issue in the literature. Notice that usually the coefficient attached to inflation is normalized to 1. According to Orphanides and Williams (2006), the coefficient attached to the output gap is set to 4, in line with Okun's law. This implies equal weights to the rate of inflation and the output gap in the loss function. Orphanides and Williams (2006) also consider a benchmark calibration $\varphi^{r}=1$. In the remainder of the paper, we will set $\varphi^{q}=4$. However, our qualitative results are not affected if we consider smaller values for the relative weight attached to the interest rate smoothing term. Overall, our results are quite robust across different calibrations of the coefficients characterizing the relative importance of competing welfare objectives.

A word of caution is in order at this stage. In a standard NK setting it is possible to provide microfoundations for a loss function that balances inflation and output gap variability through a second order approximation (see Woodford, 2003). Conversely, in the present framework there is no direct analytical rationale for the introduction of a term penalizing stock price fluctuations [as in (38) and (39)]. The purpose of this exercise is to assess the desirability to react to asset price fluctuations in the presence of a cost channel effect.

In the remainder of this section numerical results are presented just under loss specifications (36) and (39). Moreover, a perfect pass-through from policy to bank lending rates $(\psi=0)$ is assumed in the graphical analysis. ${ }^{11}$ Figures 12 and 13 report the loss under different combinations of $\chi_{y}$ and $\chi_{\pi}$ under the contemporaneous data rule, while Figures 14 and 15 refer to the forward looking rule. In addition, Tables 1 and 2 report the minimum loss and the corresponding response parameters to inflation and output gap, both under the contemporaneous (28) and the forward looking rule (32). ${ }^{12}$ Moreover, we report the loss corresponding to a benchmark

\footnotetext{
${ }^{10}$ To counter this criticism, they incorporate sticky wages in the basic model and show that indeterminacy may still arise even if profits are now affected by both sources of nominal rigidity.

${ }^{11}$ Notice that the contour maps are plotted only in correspondence with the space of determinacy.

${ }^{12}$ Notice that, as in the graphical inspection, we consider a maximum response of 4 in the $\left\{\chi_{\pi}, \chi_{y}\right\}$ space. Therefore, the concept of minimum loss has to be regarded with respect to the space considered.
} 
parameterization often implemented in numerical studies, namely $\chi_{\pi}=1.5$ and $\chi_{y}=0.5$. The results are conditioned to the degree of pass-through $(\psi=\{-1,0,0.5\})$ and to the response to asset prices, which is either set to 0 or to 0.1 .
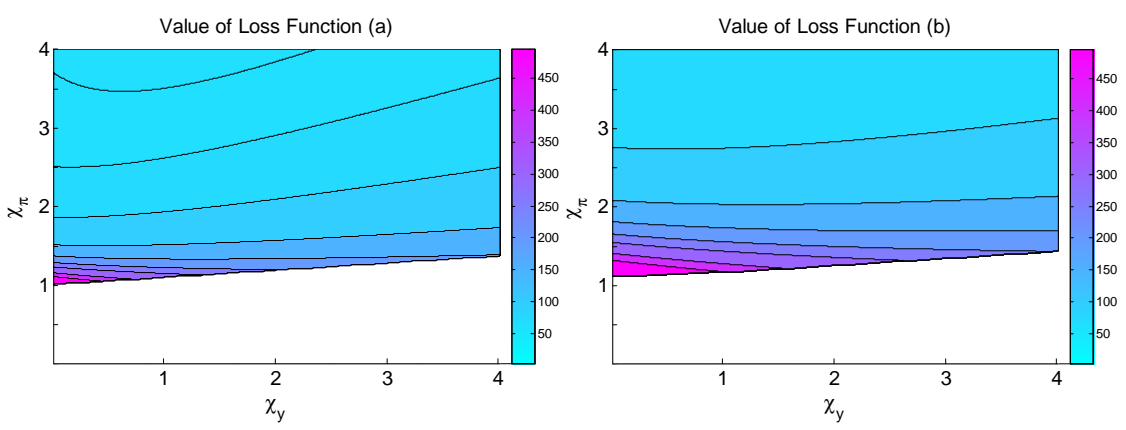

Figure 12: Loss for contemporaneous data rule in the region of determinacy under (36):

(a) $\chi_{q}=0$ and (b) $\chi_{q}=0.1$
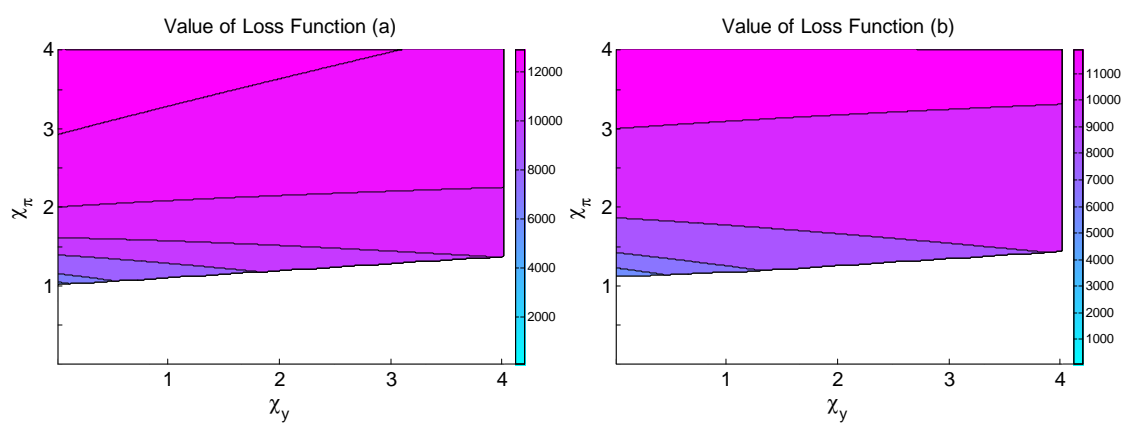

Figure 13: Loss for contemporaneous data rule in the region of determinacy under (39):

$$
\text { (a) } \chi_{q}=0 \text { and (b) } \chi_{q}=0.1
$$

Figure 12 shows that, under (36), welfare losses are generally smaller if the central bank is strenuously involved in keeping inflation under control. At the same time, reacting to asset price misalignments exerts a negative impact. In Figure 13, we report the results under the loss function (39). Previous evidence is reversed when the central bank cares about asset prices variability. In this case the loss is on average smaller if the monetary authority attaches a low reaction coefficient to the rate of inflation.

Figures 14 and 15 plot the contour map for the loss functions (36) and (39) under a forward looking interest rate rule. The graphical inspection suggests that the results under a forward looking rule are qualitatively assimilable to those detailed for a contemporaneous data rule. Moreover, numerical analysis suggests that, in the presence of any institutional or operational reason to be concerned with asset prices variability, then the central bank is better off by responding to asset prices misalignments. 
Loss under (36)

\begin{tabular}{c|ccc}
\hline \multicolumn{1}{c}{ Min Loss } & Min at $\left\{\chi_{\pi}, \chi_{y}\right\}$ & Loss at $\chi_{\pi}=1.5$ and $\chi_{y}=0.5$ \\
\hline \hline$\psi=-1 ; \chi_{q}=0$ & 52.2 & $\{4,2.42\}$ & 120.1 \\
$\psi=-1 ; \chi_{q}=0.1$ & 61.9 & $\{4,3.37\}$ & 223.5 \\
$\psi=0 ; \chi_{q}=0$ & 64.1 & $\{4,0.95\}$ & 149.1 \\
$\psi=0 ; \chi_{q}=0.1$ & 74.9 & $\{4,0.75\}$ & 288.6 \\
$\psi=0.5 ; \chi_{q}=0$ & 73.6 & $\{4,0.37\}$ & 177.5 \\
$\psi=0.5 ; \chi_{q}=0.1$ & 83.9 & $\{4,0\}$ & 352.1 \\
\hline \multicolumn{4}{c}{ Loss under $(39)$} \\
\hline$\psi=-1 ; \chi_{q}=0$ & 3361 & $\{1,0.01\}$ & 8276 \\
$\psi=-1 ; \chi_{q}=0.1$ & 2809 & $\{1.05,0.02\}$ & 6400 \\
$\psi=0 ; \chi_{q}=0$ & 5819 & $\{1,0\}$ & 10718 \\
$\psi=0 ; \chi_{q}=0.1$ & 5416 & $\{1.11,0\}$ & 8871 \\
$\psi=0.5 ; \chi_{q}=0$ & 8042 & $\{1,0\}$ & 12574 \\
$\psi=0.5 ; \chi_{q}=0.1$ & 8090 & $\{1.15,0\}$ & 10914 \\
\hline \hline
\end{tabular}

Table 2: Loss under contemporaneous data rule
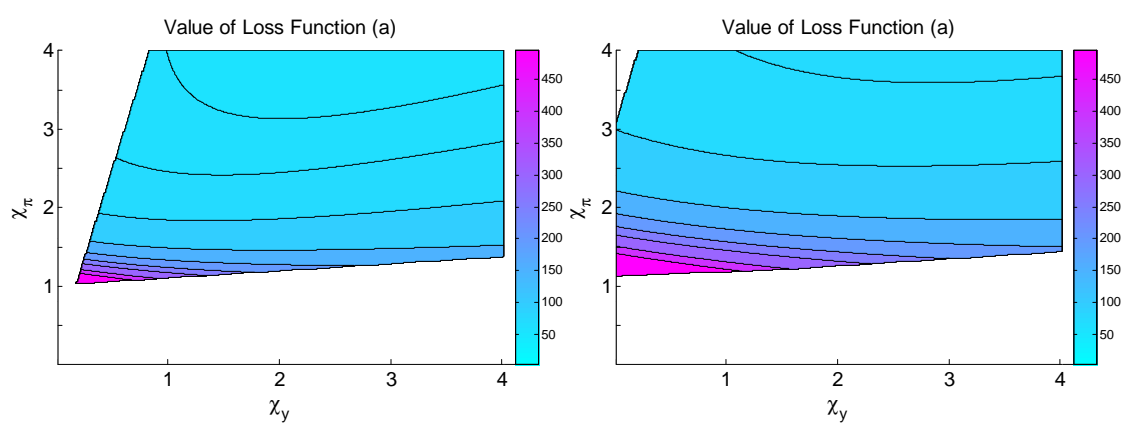

Figure 14: Loss for forward looking rule in the region of determinacy under (36):

(a) $\chi_{q}=0$ and (b) $\chi_{q}=0.1$
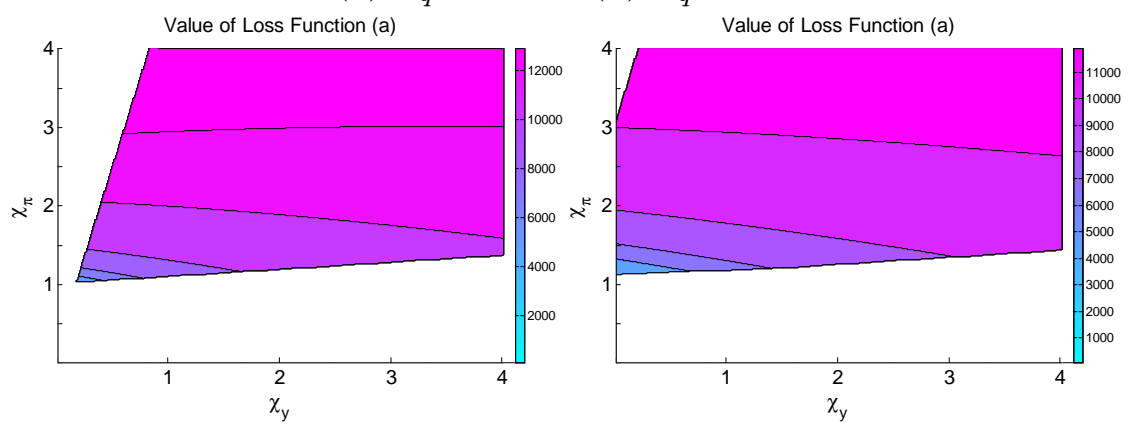

Figure 15: Loss for forward looking rule in the region of determinacy under (39):

$$
\text { (a) } \chi_{q}=0 \text { and (b) } \chi_{q}=0.1
$$

We can observe that loss under both specifications (36) and (39) increases in the degree of pass-through. Reacting to asset prices misalignments determines an increase in the overall volatility, at every value of the pass-through. Not surprisingly, the inclusion of the asset price volatility term into the loss function determines a marked increase in the overall welfare loss. This can be ascribed to the presence of excess volatility in asset prices misalignments, compared to inflation and output gap. Excess volatility is an inherent feature of asset prices and it is often 
Loss under (36)

\begin{tabular}{|c|c|c|c|}
\hline & Min Loss & Min at $\left\{\chi_{\pi}, \chi_{y}\right\}$ & Loss at $\chi_{\pi}=1.5$ and $\chi_{y}=0.5$ \\
\hline$\psi=-1 ; \chi_{q}=0$ & "47.3 & (24,4\} & 124.1 \\
\hline$\psi=-1 ; \chi_{q}=0.1$ & 53.6 & $\{4,4\}$ & 238.0 \\
\hline$\psi=0 ; \chi_{q}=0$ & 63.3 & $\{4,2.81\}$ & 157.4 \\
\hline$\psi=0 ; \chi_{q}=0.1$ & 70.9 & $\{4,3.1\}$ & 319.3 \\
\hline$\psi=0.5 ; \chi_{q}=0$ & 73.5 & $\{4,1.94\}$ & 190.3 \\
\hline$\psi=0.5 ; \chi_{q}=0.1$ & 83.9 & $\{4,1.84\}$ & 398.5 \\
\hline \multicolumn{4}{|c|}{ Loss under (39) } \\
\hline$\psi=-1 ; \chi_{q}=0$ & 2377 & $\{1,0.01\}$ & 8131 \\
\hline$\psi=-1 ; \chi_{q}=0.1$ & 2131 & $\{1.05,0.02\}$ & 6165 \\
\hline$\psi=0 ; \chi_{q}=0$ & 5113 & $\{1.02,0.17\}$ & 10505 \\
\hline$\psi=0 ; \chi_{q}=0.1$ & 4465 & $\{1.12,0\}$ & 8545 \\
\hline$\psi=0.5 ; \chi_{q}=0$ & 8073 & $\{1.05,0.29\}$ & 12344 \\
\hline$\psi=0.5 ; \chi_{q}=0.1$ & 7212 & $\{1.17,0\}$ & 10570 \\
\hline
\end{tabular}

Table 3: Loss under forward looking rule

advanced as a rationale for avoiding an explicit response to them (see, e.g., Bullard and Schaling, 2002). In addition, our numerical exercises confirm that it is beneficial to react to asset prices misalignments when the loss function incorporates a term reflecting their volatility, for both Taylor-type rules and for both criteria of welfare loss evaluation.

The minimum possible loss is generally lower under a forward looking rule than under a contemporaneous data reaction function. However, the minimum loss with a forward looking rule under (36) is often achieved at "implausibly" high responses to output gap. The advantage of the forward looking rule over the contemporaneous rule in terms of minimum loss tends to vanish as the degree of pass-through increases. Therefore, we find quite informative to assess the loss under a more plausible parameterization, such as $\left\{\chi_{\pi}=1.5, \chi_{y}=0.5\right\}$. In this case, we can notice that a contemporaneous data rule produces a lower loss, both under (36) and (39).

Moreover, as to the combination of $\left\{\chi_{\pi}, \chi_{y}\right\}$ ensuring the minimum loss, a strong reaction to inflation is generally required under (36), while $\chi_{y}$ is lower and decreases in the degree of passthrough. Intuitively, as the pass-through parameter increases, reflecting a financial accelerator effect via the banking sector, the volatilities of inflation, asset prices and output gap rise. We find that output gap variability has a relatively small response to changes in $\psi$, compared to that of inflation and asset prices. ${ }^{13}$ This effect is even more evident under (39). When the loss function incorporates a term reflecting asset prices volatility, the monetary authority needs a relatively higher $\chi_{q}$. In this case, also the response to inflation must be minimal, as asset prices volatility accounts for the majority of the welfare loss. In addition, further numerical analysis shows that asset prices volatility increases in $\chi_{\pi}$ and $\chi_{y}$.

\footnotetext{
${ }^{13}$ Notice that the degree of pass-through does not enter the IS specification.
} 


\section{Concluding Remarks}

We develop a New Keynesian macroeconomic model to study the interplay between capital market imperfections, firm profitability and inflation dynamics. We rely on a sticky price framework featuring a cost-side effect along the lines traced by Ravenna and Walsh (2006). We extend the baseline model in two main directions: first, following Chowdhury, Hoffmann, and Schabert (2006), we allow for the introduction of varying degrees of interest rate changes to affect firms' costs of lending; second, we consider the direct influence of credit market distortions on firm profitability and, in turn, stock price dynamics. We provide an analytical treatment of the conditions ensuring REE uniqueness and E-stability when the monetary authority reacts only to inflation. Standard conditions turn out to be significantly different in the presence of strong credit market distortions.

Numerical simulations allow us to study the performance of interest rate rules reacting to the current (or expected) output gap, inflation and asset prices misalignments. Moreover, we assess the loss of welfare incurred by the monetary authority in the presence of different degrees of the pass-through between the policy instrument and bank lending rates under different Taylortype rules. Our analysis shows that considering a one-to-one relationship between policy rates and bank lending rates, as assumed in Ravenna and Walsh (2006), can be quite misleading for the sake of designing a rule that ensures REE determinacy and stability under adaptive learning when the cost channel matters. We show that in conditions of uncertainty regarding the degree of pass-through between policy and bank lending rates, the central bank is generally better off following a rule in which the policy instrument is set according to misalignments in contemporaneous data rather than adopting an expectational interest rate rule. Moreover, in the former case, a considerably wider area of determinacy can be achieved. In addition, for a plausible range of responses to inflation and output gap the adoption of a contemporaneous data rule ensures, on average, a lower welfare loss.

When the cost channel matters, the risk of indeterminacy and instability under adaptive learning can be reduced if the central bank reacts positively to actual (or expected) asset prices misalignments from their frictionless values or to current (or expected) output gap. It can often be argued that a response to asset prices might be even more desirable than a response to the output gap to ensure determinacy. This is the case when monetary policy is conducted in environments characterized by strong credit market distortions. In principle, this strategy has a negative effect on firms' profitability and raises the risk of inflationary pressures. Nevertheless, as in a sticky price framework firms' profitability is negatively related to the output gap, inflationary pressures brought by the cost channel effect can be smoothed down if the central bank responds positively to asset prices misalignments. Therefore, a positive response to out-ofequilibrium dynamics in asset prices balances the negative impact brought by the cost channel on inflation dynamics, especially in the presence of a strong pass-through from policy to bank lending rates. These results contradict the policy prescriptions achieved by previous studies exploring the role of monetary policy as a potential financial stabilizer. Most importantly, our findings highlight the need to carefully assess the degree of credit market distortions to draw 
sound prescriptions for monetary policy design.

Future work appears desirable in several areas. In a companion paper, we explore the implications of the cost channel for the design of optimal monetary policy, assuming that the central bank is faced with a loss function that balances asset price variability along with output and inflation. We also aim at exploring further the nature of the E-stable equilibria in the area of indeterminacy, in order to assess the conditions for sunspots learnability and their policy implications. More generally, we argue that it is important to further explore the informational content of asset prices for the conduct of monetary policy. 


\section{REFERENCES}

Airaudo, M., S. Nisticò, And L.-F. Zanna (2006): "Learning, Asset Prices and Monetary Policy," Mimeo, Collegio Carlo Alberto.

Barth, M. J., And V. A. Ramey (2000): "The Cost Channel of Monetary Transmission," NBER Working Papers 7675, National Bureau of Economic Research, Inc.

Bernanke, B., and M. Gertler (1989): "Agency Costs, Net Worth, and Business Fluctuations," American Economic Review, 79(1), 14-31.

(1990): "Financial Fragility and Economic Performance," The Quarterly Journal of Economics, 105(1), 87-114.

(1999): "Monetary policy and asset price volatility," Economic Review, (Q IV), 17-51.

Bernanke, B. S., and M. Gertler (2001): "Should Central Banks Respond to Movements in Asset Prices?," American Economic Review, 91(2), 253-257.

Bernanke, B. S., M. Gertler, and S. Gilchrist (1999): "The financial accelerator in a quantitative business cycle framework," in Handbook of Macroeconomics, ed. by J. B. Taylor, and M. Woodford, vol. 1 of Handbook of Macroeconomics, chap. 21, pp. 1341-1393. Elsevier.

Blanchard, O. J., and C. M. Kahn (1980): "The Solution of Linear Difference Models under Rational Expectations," Econometrica, 48(5), 1305-11.

BrÜCKner, M., and A. Schabert (2003): "Supply-side effects of monetary policy and equilibrium multiplicity," Economics Letters, 79(2), 205-211.

Bullard, J., And K. Mitra (2002): "Learning about monetary policy rules," Journal of Monetary Economics, 49(6), 1105-1129.

Bullard, J. B., And E. Schaling (2002): "Why the Fed should ignore the stock market," Review, (Mar.), 35-42.

Calvo, G. A. (1983): "Staggered prices in a utility-maximizing framework," Journal of Monetary Economics, 12(3), 383-398.

Carlstrom, C. T., and T. Fuerst (2007): "Asset Prices, Nominal Rigidities, and Monetary Policy," Review of Economic Dynamics, 10(2), 256-275.

Chowdhury, I., M. Hoffmann, and A. Schabert (2006): "Inflation dynamics and the cost channel of monetary transmission," European Economic Review, 50(4), 995-1016.

Christiano, L. J., M. Eichenbaum, and C. L. Evans (1997): "Sticky price and limited participation models of money: A comparison," European Economic Review, 41(6), 12011249. 
Clarida, R., J. Gali, and M. Gertler (1999): "The Science of Monetary Policy: A New Keynesian Perspective," Journal of Economic Literature, 37(4), 1661-1707.

Eichenbaum, M. (1992): "Interpreting the macroeconomic time series facts: The effects of monetary policy' : by Christopher Sims," European Economic Review, 36(5), 1001-1011.

Evans, G. W., And S. Honkapohja (2001): Learning and Expectations in Macroeconomics. Princeton University Press.

Faia, E., and T. Monacelli (2007): "Optimal interest rate rules, asset prices, and credit frictions," Journal of Economic Dynamics and Control, 31(10), 3228-3254.

Galì, J., And M. Gertler (1999): "Inflation dynamics: A structural econometric analysis," Journal of Monetary Economics, 44(2), 195-222.

Gandolfo, G. (1996): Economic Dynamics. Springer-Verlag, 3rd edn. edn.

Genberg, H., J. Lipsky, S. Cecchetti, and S. Wadhwani (2000): Asset Prices and Central Bank Policy. Centre For Economic Policy Research and International Center For Monetary And Banking Studies.

Gilchrist, S., and M. Saito (2006): "Expectations, Asset Prices, and Monetary Policy: The Role of Learning," NBER Working Papers 12442, National Bureau of Economic Research, Inc.

Greenwald, B. C., and J. E. Stiglitz (1988): "Imperfect Information, Finance Constraints and Business Fluctuations," in Finance Constraints, Expectations and Macroeconomics, ed. by M. Kohn, and S. Tsiang, pp. 103-140. Oxford University Press.

(1993): "Financial Market Imperfections and Business Cycles," The Quarterly Journal of Economics, 108(1), 77-114.

Hannan, T. H., and A. N. Berger (1991): "The Rigidity of Prices: Evidence from the Banking Industry," American Economic Review, 81(4), 938-45.

Hülsewig, O., E. Mayer, and T. Wollmershäuser (2006): "Bank Behavior and the Cost Channel of Monetary Transmission," CESifo Working Paper Series 1813, CESifo GmbH.

Ireland, P. N. (2004): "Technology Shocks in the New Keynesian Model," The Review of Economics and Statistics, 86(4), 923-936.

Kiyotaki, N., And J. Moore (1997): "Credit Cycles," Journal of Political Economy, 105(2), $211-48$.

Llosa, G., and V. Tuesta (2007): "E-stability of Monetary Policy when the Cost Channel Matters," Mimeo, Central Reserve Bank of Peru. 
McCallum, B. T., and E. Nelson (1999): "Performance of Operational Policy Rules in an Estimated Semi-Classical Model," in Monetary Policy Rules, ed. by J. Taylor, pp. 15-45. University of Chicago Press.

Myers, S. C., ANd N. S. Majluf (1984): "Corporate financing and investment decisions when firms have information that investors do not have," Journal of Financial Economics, $13(2), 187-221$.

Nisticò, S. (2006): "Monetary Policy and Stock-Price Dynamics in a DSGE Framework," in Monetary Policy, Banks and Institutions, ed. by G. D. Giorgio, and F. Neri. Luiss University Press, Rome.

Orphanides, A., and J. C. Williams (2006): "Inflation targeting under imperfect knowledge," Finance and Economics Discussion Series 2006-20, Board of Governors of the Federal Reserve System (U.S.).

Ravenna, F., and C. E. Walsh (2006): "Optimal monetary policy with the cost channel," Journal of Monetary Economics, 53(2), 199-216.

Sbordone, A. M. (2002): "Prices and unit labor costs: a new test of price stickiness," Journal of Monetary Economics, 49(2), 265-292.

Sims, C. A. (1992): "Interpreting the macroeconomic time series facts : The effects of monetary policy," European Economic Review, 36(5), 975-1000.

Smets, F., And R. Wouters (2003): "An Estimated Dynamic Stochastic General Equilibrium Model of the Euro Area," Journal of the European Economic Association, 1(5), 1123-1175.

Steinsson, J. (2003): "Optimal monetary policy in an economy with inflation persistence," Journal of Monetary Economics, 50(7), 1425-1456.

Walsh, C. E. (2003): Monetary Theory and Policy. The MIT Press, second edn.

Woodford, M. (1999): "Optimal Monetary Policy Inertia," Manchester School, 67(0), 1-35.

(2003): Interest and Prices: Foundations of a Theory of Monetary Policy. Princeton University Press. 


\section{Appendix A}

Steady State Values

$$
\begin{array}{ccc}
\pi=0 & 1=\beta R & C=C_{j}=N=N_{j}=Y=W^{\frac{1}{\eta+\sigma}} \\
Z=1 & A=1 & H=1 \\
D=Y(1-\Phi) & \frac{M}{P}=\left(C^{\sigma} \frac{R}{R-1}\right)^{\frac{1}{\tau}} & \Pi^{\text {int }}=0 \\
Q=\frac{D}{1-\beta} & \frac{M^{d}}{P}=W L & W=\frac{\beta}{\Phi} .
\end{array}
$$

\subsection{E-stability: Contemporaneous rule.}

Proposition 6. Under a contemporaneous data interest rate rule responding to the rate of inflation, the following conditions ensuring E-stability can be identified:

- Iff $\psi>-1$

$$
\chi_{\pi}>\bar{\chi}_{\pi}=1
$$

- Iff $\psi<1+\frac{2 \eta}{\sigma}$ :

$$
\chi_{\pi}>\widehat{\chi}_{\pi}=\frac{(1-\kappa) \sigma-\sigma \beta-\kappa \eta}{(\sigma(\psi-1)-2 \eta) \kappa}
$$

- Iff $\psi>1+\frac{2 \eta}{\sigma}$ :

$$
\chi_{\pi}<\widetilde{\chi}_{\pi}=\frac{(1-\kappa) \sigma-\sigma \beta-\kappa \eta}{(\sigma(\psi-1)-2 \eta) \kappa}
$$

Proof. If we set $\chi_{q}=0$ in (28), the discount factor $\beta$ turns out to be one of the three eigenvalues of the matrix $\boldsymbol{\Gamma}_{c}^{-1} \boldsymbol{\Omega}_{c}$. Under these settings, the NK Phillips curve and the IS curve constitute an autonomous system. Moreover, when $\chi_{y}=0$ the matrix of structural parameters associated to the forward looking vector is the following cofactor:

$$
\mathbf{J}_{c 33}=\left[\begin{array}{cc}
\frac{\kappa \sigma+\sigma \beta+\kappa \eta}{\sigma+\kappa \eta \chi_{\pi}-\kappa \sigma \psi \chi_{\pi}}-1 & \frac{\kappa \sigma^{2}+\kappa \eta \sigma}{\sigma+\kappa \eta \chi_{\pi}-\kappa \sigma \psi \chi_{\pi}} \\
-\frac{\kappa \chi_{\pi}+\beta \chi_{\pi}+\kappa \psi \chi_{\pi}-1}{\sigma+\kappa \eta \chi_{\pi}-\kappa \sigma \psi \chi_{\pi}} & -\frac{\kappa \sigma \chi_{\pi}-\sigma+\kappa \sigma \psi \chi_{\pi}}{\sigma+\kappa \eta \chi_{\pi}-\kappa \sigma \psi \chi_{\pi}}-1
\end{array}\right] .
$$

This setup implies that the necessary and sufficient conditions guaranteeing E-stability can be stated as follows:

$$
\begin{aligned}
& B_{c}>0 \\
& A_{c}>0,
\end{aligned}
$$

where $A_{c}$ and $B_{c}$ are the coefficients of the characteristic polynomial retrievable from $\mathbf{J}_{c 33}-\mathbf{I}$ :

$$
\lambda^{2}+A_{c} \lambda+B_{c}=0
$$


Thus:

$$
\begin{aligned}
& A_{c}=\frac{\kappa \sigma \chi_{\pi}-\sigma+\kappa \sigma \psi \chi_{\pi}-\kappa \sigma+\sigma \beta+\kappa \eta}{\sigma+\kappa \eta \chi_{\pi}-\kappa \sigma \psi \chi_{\pi}}+2 \\
& B_{c}=\frac{\left(\kappa \sigma^{2}+\kappa \eta \sigma\right)\left(\kappa \chi_{\pi}+\beta \chi_{\pi}+\kappa \psi \chi_{\pi}-1\right)}{\left(\sigma+\kappa \eta \chi_{\pi}-\kappa \sigma \psi \chi_{\pi}\right)^{2}}-\left(\frac{\kappa \sigma \chi_{\pi}-\sigma+\kappa \sigma \psi \chi_{\pi}}{\sigma+\kappa \eta \chi_{\pi}-\kappa \sigma \psi \chi_{\pi}}+1\right)\left(\frac{\kappa \sigma+\sigma \beta+\kappa \eta}{\sigma+\kappa \eta \chi_{\pi}-\kappa \sigma \psi \chi_{\pi}}-1\right)
\end{aligned}
$$

Let us focus on condition (43):

$$
A_{c}=\frac{\kappa \sigma \chi_{\pi}-\sigma+\kappa \sigma \psi \chi_{\pi}-\kappa \sigma+\sigma \beta+\kappa \eta}{\sigma+\kappa \chi_{\pi}(\eta-\sigma \psi)}+2>0
$$

We have to multiply both sides by the term $\sigma+\kappa \chi_{\pi}(\eta-\sigma \psi)$. On the one hand, this factor is always positive for $\psi<\frac{\eta}{\sigma}$ (sufficient condition). On the other hand, when $\psi>\frac{\eta}{\sigma}$, we need to introduce a restriction on $\kappa$ in order to ensure its positiveness.

Condition 1: In order to rule out the possibility that the term $\sigma+\kappa \chi_{\pi}(\eta-\sigma \psi)$ is negative, we have to assume that iff $\psi>\frac{\eta}{\sigma}$ the following restriction applies:

$$
0<\kappa<\frac{\sigma}{\chi_{\pi}(\sigma \psi-\eta)}
$$

Notice that this condition holds across different plausible parameterizations and is always satisfied by the three sets of calibrated parameters taken into consideration in the paper. Thus, we can rearrange (43) as:

$$
(\kappa-1) \sigma+\sigma \beta+\kappa \eta+(\sigma(\psi-1)-2 \eta) \kappa \chi_{\pi}>0
$$

At this stage there are two relevant cases to consider, namely $1+\frac{2 \eta}{\sigma}<\psi$ and $1+\frac{2 \eta}{\sigma}>\psi$. In the first case, the response to inflation must satisfy the following condition:

$$
\chi_{\pi}>\frac{(1-\kappa) \sigma-\sigma \beta-\kappa \eta}{(\sigma(\psi-1)-2 \eta) \kappa}
$$

Otherwise, the maximum response to inflation is constrained from the following condition:

$$
\chi_{\pi}<\frac{(1-\kappa) \sigma-\sigma \beta-\kappa \eta}{(\sigma(\psi-1)-2 \eta) \kappa}
$$

Let us focus now on (44):

$$
\frac{\kappa\left(\chi_{\pi}-1\right)(\sigma+\eta)}{\sigma+\kappa \chi_{\pi}(\eta-\sigma \psi)}>0
$$

as we assume that denominator is always positive, (45) always holds for $\chi_{\pi}>1$.

Corollary 7. The set of conditions stated in the proposition above allows us to determine an interval for critical values of the pass-through:

1. Iff $\psi<\frac{\eta}{\sigma}+\frac{(1-\beta)}{\kappa}$ the system will always be E-stable if $\chi_{\pi}>1$. 
2. Iff $\frac{\eta}{\sigma}+\frac{(1-\beta)}{\kappa} \leq \psi<1+\frac{2 \eta}{\sigma}$ the response coefficient to inflation has to be set according to $\chi_{\pi}>\widehat{\chi}_{\pi}$

3. Iff $\psi \geq 1+\frac{2 \eta}{\sigma}$ E-stability is never attained.

Proof. Notice that conditions (40) and (41) represent a minimum response threshold. The conditions reported in Proposition 6 are considered in the $\left\{\chi_{\pi}, \psi\right\}$ space.

By equating (40) and (41) we find the value of $\psi$ from which the condition (41) is binding. From the point where conditions (41) and (42) equal $\left(\psi=1+\frac{2 \eta}{\sigma}\right)$ E-stability can never be attained.

\subsection{E-stability: forward looking rule.}

Proposition 8. Under an interest rate rule responding only to expected inflation in the presence of a cost channel, the necessary and sufficient conditions guaranteeing E-stability can be stated as follows:

- Iff $\psi>-1$ :

$$
\chi_{\pi}>\bar{\chi}_{\pi}=1
$$

- Iff $\psi<\frac{\eta}{\sigma}$ :

$$
\chi_{\pi}>\widehat{\chi}_{\pi}=\frac{\kappa(\sigma+\eta)-\sigma(1-\beta)}{\kappa(\eta-\sigma \psi)}
$$

- Iff $\psi>\frac{\eta}{\sigma}$ :

$$
\chi_{\pi}<\tilde{\chi}_{\pi}=\frac{\sigma(1-\beta)-\kappa(\sigma+\eta)}{\kappa(\sigma \psi-\eta)}
$$

Proof. For $\chi_{q}=\chi_{y}=0$ the matrix of structural parameters associated to the forward looking vector is represented by the following cofactor:

$$
\mathbf{J}_{f 33}=\left[\begin{array}{cc}
\beta+\kappa \chi_{\pi}(\psi+1)-\frac{\kappa}{\sigma}\left(\chi_{\pi}-1\right)(\sigma+\eta) & \kappa(\sigma+\eta) \\
-\frac{1}{\sigma}\left(\chi_{\pi}-1\right) & 1
\end{array}\right] .
$$

The necessary and sufficient conditions guaranteeing E-stability are:

$$
\begin{aligned}
& B_{f}>0 \\
& A_{f}>0
\end{aligned}
$$

where are the parameters of the characteristic polynomial associated to $\mathbf{J}_{f 33}-\mathbf{I}$. Thus (49) and (50) read as:

$$
\begin{aligned}
\left(\frac{\kappa}{\sigma}\left(\chi_{\pi}-1\right)(\sigma+\eta)-\kappa \chi_{\pi}(\psi+1)-\beta+1\right) & >0 \\
\frac{\kappa}{\sigma}\left(\chi_{\pi}-1\right)(\sigma+\eta) & >0
\end{aligned}
$$


It is immediate to verify that $A_{f}$ is always greater than zero iff $\chi_{\pi}>1$. As to condition (??), this can be rearranged as:

$$
(\eta-\sigma \psi) \kappa \chi_{\pi}>\kappa(\sigma+\eta)-\sigma(1-\beta)
$$

Now, iff $\psi<\frac{\eta}{\sigma}$, the relevant condition reads as:

$$
\chi_{\pi}>\frac{\kappa(\sigma+\eta)-\sigma(1-\beta)}{\kappa(\eta-\sigma \psi)}
$$

otherwise, for $\psi>\frac{\eta}{\sigma}$ :

$$
\chi_{\pi}<\frac{\sigma(1-\beta)-\kappa(\sigma+\eta)}{\kappa(\sigma \psi-\eta)}
$$

Corollary 9. The set of conditions stated in the proposition above allows us to determine an interval for critical values of the pass-through:

1. Iff $\psi<\frac{1-\kappa-\beta}{\kappa}$ the system will always be E-stable if $\chi_{\pi}>1$.

2. Iff $\frac{1-\kappa-\beta}{\kappa} \leq \psi<\frac{\eta}{\sigma}$ the response coefficient to inflation has to be set according to $\chi_{\pi}>\widehat{\chi}_{\pi}$;

3. Iff $\psi \geq \frac{\eta}{\sigma}$ E-stability is never attained.

Proof. Notice that conditions (46) and (47) represent a minimum response threshold. The conditions reported in Proposition 8 are considered in the $\left\{\chi_{\pi}, \psi\right\}$ space.

By equating (46) and (47) we find the value of $\psi$ from which the condition (47) is binding. From the point where conditions (47) and (48) equal $\left(\psi=\frac{\eta}{\sigma}\right)$ E-stability can never be attained.

6.3. Proof Proposition 2. Proof. If we set $\chi_{q}=0$ in (28), the discount factor $\beta$ turns out to be one of the three eigenvalues of the matrix $\boldsymbol{\Gamma}_{c}^{-1} \boldsymbol{\Omega}_{c}$. Under these settings, the NK Phillips curve and the IS curve constitute an autonomous system, where the matrix of structural parameters associated to the forward looking vector is the following cofactor:

$$
\mathbf{J}_{c 33}=\left[\begin{array}{cc}
\frac{\sigma \beta+\kappa \sigma+\kappa \eta}{\sigma+\kappa \eta \chi_{\pi}-\kappa \sigma \psi \chi_{\pi}} & \frac{\kappa \sigma^{2}+\kappa \eta \sigma}{\sigma+\kappa \eta \chi_{\pi}-\kappa \sigma \psi \chi_{\pi}} \\
-\frac{\kappa \chi_{\pi}-1+\kappa \psi \chi_{\pi}+\beta \chi_{\pi}}{\sigma+\kappa \eta \chi_{\pi}-\kappa \sigma \psi \chi_{\pi}} & -\frac{\kappa \sigma \chi_{\pi}-\sigma+\kappa \sigma \psi \chi_{\pi}}{\sigma+\kappa \eta \chi_{\pi}-\kappa \sigma \psi \chi_{\pi}}
\end{array}\right] .
$$

This setup implies that the necessary and sufficient conditions guaranteeing determinacy can be stated as follows:

$$
\begin{aligned}
& \left|B_{c}\right|<1 \\
& \left|A_{c}\right|<1+B_{c}
\end{aligned}
$$

where $A_{c}$ and $B_{c}$ are the coefficients of the characteristic polynomial retrievable from $\mathbf{J}_{c 33}$ :

$$
\lambda^{2}+A_{c} \lambda+B_{c}=0 .
$$


Assuming that $\chi_{y}=0$ we obtain:

$$
\begin{aligned}
& A_{c}=\frac{\kappa \sigma \chi_{\pi}(1+\psi)-\sigma(1+\beta)-\kappa(\sigma+\eta)}{\sigma+\kappa \chi_{\pi}(\eta-\sigma \psi)}, \\
& B_{c}=\frac{\beta \sigma}{\sigma+\sigma+\kappa \chi_{\pi}(\eta-\sigma \psi)} .
\end{aligned}
$$

Let us focus first on condition (53):

$$
\left|\frac{\beta \sigma}{\sigma+\kappa \chi_{\pi}(\eta-\sigma \psi)}\right|<1
$$

which translates into:

$$
\begin{aligned}
\frac{\beta \sigma}{\sigma+\kappa \chi_{\pi}(\eta-\sigma \psi)} & <1 \\
\frac{\beta \sigma}{\sigma+\kappa \chi_{\pi}(\eta-\sigma \psi)} & >-1 .
\end{aligned}
$$

We take into consideration the first inequality (55). We have to multiply both sides by its denominator. On the one hand, this factor is always be positive for $\psi<\frac{\eta}{\sigma}$ (sufficient condition). On the other hand, when $\psi>\frac{\eta}{\sigma}$, we need to introduce a restriction on $\kappa$ in order to ensure its positiveness. Under Condition 1, we can rearrange (55) as:

$$
\sigma(\beta-1)<\kappa \chi_{\pi}(\eta-\sigma \psi)
$$

In order to derive a condition for the response coefficient $\chi_{\pi}$ we have to divide the inequality by $\kappa(\eta-\sigma \psi)$ on both sides. This term is negative when $\sigma \psi>\eta$. In this case we end up with the following condition:

$$
\frac{\sigma(\beta-1)}{\kappa(\eta-\sigma \psi)}>\chi_{\pi}
$$

In the opposite case, when $\psi<\frac{\eta}{\sigma}$, we get:

$$
\frac{\sigma(\beta-1)}{\kappa(\eta-\sigma \psi)}<\chi_{\pi}
$$

Note that the term on the LHS of (58) is always negative. Let us consider condition (56):

$$
-\sigma(\beta+1)<\kappa \chi_{\pi}(\eta-\sigma \psi) .
$$

Again, in order to isolate $\chi_{\pi}$ on the RHS we have to divide both sides of the inequality by $\kappa(\eta-\sigma \psi)$. Thus, iff $\psi>\frac{\eta}{\sigma}$, we get:

$$
\frac{\sigma(\beta+1)}{\kappa(\sigma \psi-\eta)}>\chi_{\pi}
$$

When $\psi>\frac{\eta}{\sigma}$ the term $\frac{\sigma(\beta+1)}{\kappa(\sigma \psi-\eta)}$ is always positive under the restriction characterizing the baseline 
parameterization. In the opposite case when $\psi<\frac{\eta}{\sigma}$ we get:

$$
\frac{\sigma(\beta+1)}{\kappa(\sigma \psi-\eta)}<\chi_{\pi}
$$

As this threshold is always be negative, condition (60) is fully nested in $\chi_{\pi}>1$. Thus we do not have to consider it.

Let us turn the attention to the second condition for determinacy, namely (54). This leads to the following inequalities:

$$
\begin{aligned}
& \frac{\kappa \sigma \chi_{\pi}(1+\psi)-\sigma(1+\beta)-\kappa(\sigma+\eta)}{\sigma+\kappa \chi_{\pi}(\eta-\sigma \psi)}<1+\frac{\beta \sigma}{\sigma+\kappa \chi_{\pi}(\eta-\sigma \psi)}, \\
& \frac{\kappa \sigma \chi_{\pi}(1+\psi)-\sigma(1+\beta)-\kappa(\sigma+\eta)}{\sigma+\kappa \chi_{\pi}(\eta-\sigma \psi)}>-1-\frac{\beta \sigma}{\sigma+\kappa \chi_{\pi}(\eta-\sigma \psi)} .
\end{aligned}
$$

We first focus on the former. Again, by assuming that Condition 1 holds true we get:

$$
\chi_{\pi} \kappa(\sigma(1+2 \psi)-\eta)<2 \sigma(1+\beta)+\kappa(\sigma+\eta) .
$$

Thus we have to consider the sign of the term $\kappa(\sigma(1+2 \psi)-\eta)$. This turns out to be always positive iff $\psi<\frac{\eta}{\sigma}$ (sufficient condition). Nevertheless, we the following condition has also to be considered:

$$
\psi>\frac{\eta}{2 \sigma}-\frac{1}{2}=\frac{\eta-\sigma}{2 \sigma} .
$$

As $\frac{\eta-\sigma}{2 \sigma}<\frac{\eta}{\sigma}$ the following condition is only valid for, $\psi>\frac{\eta-\sigma}{2 \sigma}$, iff $0<\kappa<\frac{\sigma}{\chi_{\pi}(\sigma \psi-\eta)}$ :

$$
\chi_{\pi}<\frac{2 \sigma(1+\beta)+\kappa(\sigma+\eta)}{\kappa(\sigma(1+2 \psi)-\eta)}
$$

Otherwise, when $\psi<\frac{\eta-\sigma}{2 \sigma}$, we end up with:

$$
\chi_{\pi}>\frac{2 \sigma(1+\beta)+\kappa(\sigma+\eta)}{\kappa(\sigma(1+2 \psi)-\eta)} .
$$

But then this expression is always negative and thus it is nested in the Taylor principle, $\chi_{\pi}>1$. Let us consider now (62). By applying the same procedure as in the previous case, we end up with the Taylor principle:

$$
\chi_{\pi}>1
$$

Notice that (58) and (60) identify negative thresholds, hence they are always nested in condition (65). Therefore they are discarded. To sum up: 
- Iff $\psi>\frac{\eta}{\sigma}$ then the relevant conditions are:

$$
\begin{aligned}
& \frac{\sigma(\beta-1)}{\kappa(\eta-\sigma \psi)}>\chi_{\pi}, \\
& \frac{\sigma(\beta+1)}{\kappa(\sigma \psi-\eta)}>\chi_{\pi} .
\end{aligned}
$$

As we know that under this assumption that $\psi>\frac{\eta}{\sigma}$ :

$$
\frac{\sigma(\beta-1)}{(\eta-\sigma \psi) \kappa}<\frac{\sigma(1+\beta)}{\kappa(\sigma \psi-\eta)}
$$

The only binding condition is $(57): \frac{\sigma(\beta-1)}{\kappa(\eta-\sigma \psi)}=\widehat{\chi}_{\pi}>\chi_{\pi}$

- Iff $\psi<\frac{\eta}{\sigma}$ then both of conditions (58 and 60) are already nested in $\chi_{\pi}>1$.

- Iff $\psi>\frac{\eta-\sigma}{2 \sigma}$ then for condition $(63) \chi_{\pi}<\tilde{\chi}_{\pi}=\frac{2 \sigma(1+\beta)+\kappa(\sigma+\eta)}{\kappa(\sigma(1+2 \psi)-\eta)}$.

- Iff $\psi<\frac{\eta-\sigma}{2 \sigma}$ then for condition (65)

$$
\chi_{\pi}=\bar{\chi}_{\pi}>1
$$

Proof Corollary 3 Proof. Notice that $\bar{\chi}_{\pi}$ always represents the minimum response threshold on the relevant interval for the pass-through. Alternatively, conditions (29) and (30) represent a maximum response threshold. The conditions reported in Proposition 2 are considered in the $\left\{\chi_{\pi}, \psi\right\}$ space. Notice that all of them can be written as $\chi_{\pi} \lesseqgtr f\left(\psi^{-1}\right)$. Under this assumption, these conditions generally behave as hyperbolae in the relevant space. As we search for a maximum response threshold, we are interested in the functions lying on the right hand side of the asymptote of each curve. In this region all conditions are strictly decreasing functions.

The following inequality always holds: $\frac{\eta-\sigma}{2 \sigma}<\frac{\eta}{\sigma}$. Thus (30) will be binding first from the left. For $\psi \geq \frac{\eta}{\sigma}$ two conditions have to be fulfilled to ensure determinacy. We know that, for $\psi>\frac{\eta}{\sigma}$, if we compare conditions (29) and (59) the following inequality always holds:

$$
\frac{(\beta-1) \sigma}{(\eta-\sigma \psi) \kappa}<\frac{\sigma(1+\beta)}{\kappa(\sigma \psi-\eta)}
$$

All we have to do now is to compute the value of $\psi$ where conditions (29) and (30) cross at the point $\tilde{\chi}_{\pi}=\widehat{\chi}_{\pi}$ :

$$
\psi=\frac{\sigma(1-\beta)+\left(\kappa \eta \sigma^{-1}+\kappa+3 \beta+1\right) \eta}{\kappa \sigma+4 \sigma \beta+\kappa \eta} .
$$

We now have to compute the last threshold for the pass-through parameter above which determinacy is never attained. This occurs at $\widehat{\chi}_{\pi}=\bar{\chi}_{\pi}=1$. Straightforward computation shows that:

$$
\psi=\frac{\eta}{\sigma}-\frac{(\beta-1)}{\kappa}
$$


Proof Proposition 4 Proof. As the central bank does respond nor to asset prices misalignments neither to output gap, $\beta$ is one of the three eigenvalues of $\mathbf{J}_{f}$. Furthermore the NK Phillips curve and the IS constitute an autonomous system where the matrix of structural parameters associated to the forward looking vector is represented by the following cofactor:

$$
\mathbf{J}_{f 33}=\left[\begin{array}{cc}
\beta+\kappa \chi_{\pi}(\psi+1)-\frac{\kappa}{\sigma}\left(\chi_{\pi}-1\right)(\sigma+\eta) & \kappa(\sigma+\eta) \\
-\frac{1}{\sigma}\left(\chi_{\pi}-1\right) & 1
\end{array}\right] .
$$

Under a forward looking interest rate rule the necessary and sufficient conditions guaranteeing determinacy can be stated as follows:

$$
\begin{aligned}
& \left|B_{f}\right|<1, \\
& \left|A_{f}\right|<1+B_{f},
\end{aligned}
$$

where $A_{f}$ and $B_{f}$ are the coefficients of the characteristic polynomial associated to $\mathbf{J}_{f 33}$ :

$$
\lambda^{2}+A_{f} \lambda+B_{f}=0
$$

where

$$
\begin{aligned}
& A_{f}=\left(\frac{\kappa}{\sigma}\left(\chi_{\pi}-1\right)(\sigma+\eta)-\kappa \chi_{\pi}(\psi+1)-\beta-1\right) \\
& B_{f}=\beta+\kappa \chi_{\pi}(\psi+1)
\end{aligned}
$$

Let us focus first on condition (66):

$$
\left|\beta+\kappa \chi_{\pi}(\psi+1)\right|<1
$$

which translates into:

$$
\begin{aligned}
\beta+\kappa \chi_{\pi}(\psi+1) & <1 \\
-\beta-\kappa \chi_{\pi}(\psi+1) & <1 .
\end{aligned}
$$

Let us consider first condition (68). This inequality can be written as:

$$
\chi_{\pi}<\frac{1-\beta}{\kappa(\psi+1)} .
$$

Let us consider then condition (69):

$$
\kappa \chi_{\pi}(\psi+1)>-1-\beta
$$


Under $\psi>-1$ this can be expressed as:

$$
\chi_{\pi}>\frac{-1-\beta}{\kappa(\psi+1)}
$$

In the remainder we will notice that, being this threshold always negative, (71) is fully encompassed by $\chi_{\pi}>1$. Let us now focus on condition (67):

$$
\begin{aligned}
& \frac{\kappa}{\sigma}\left(\chi_{\pi}-1\right)(\sigma+\eta)-\kappa \chi_{\pi}(\psi+1)-\beta-1<1+\beta+\kappa \chi_{\pi}(\psi+1), \\
& \frac{\kappa}{\sigma}\left(\chi_{\pi}-1\right)(\sigma+\eta)-\kappa \chi_{\pi}(\psi+1)-\beta-1>-1-\beta-\kappa \chi_{\pi}(\psi+1) .
\end{aligned}
$$

We first consider condition (72). After some rearrangements this can be written as:

$$
\kappa \chi_{\pi}\left(\left(1+\frac{\eta}{\sigma}\right)-2(\psi+1)\right)<2+2 \beta+\kappa+\eta \frac{\kappa}{\sigma}
$$

Now we have to divide each side of then inequality by the term $\kappa\left(1+\frac{\eta}{\sigma}\right)-2 \kappa(\psi+1)$. This is always be positive for:

$$
\psi<\frac{\eta-\sigma}{2 \sigma}
$$

If we assume that the condition above holds we can determine the following constraint for the response coefficient:

$$
\chi_{\pi}<\frac{2+2 \beta+\kappa+\eta \frac{\kappa}{\sigma}}{\kappa\left(1+\frac{\eta}{\sigma}\right)-2 \kappa(\psi+1)}
$$

Otherwise, when $\psi>\frac{\eta-\sigma}{2 \sigma}$ :

$$
\chi_{\pi}>\frac{2+2 \beta+\sigma+\eta}{\kappa\left(1+\frac{\eta}{\sigma}\right)-2 \kappa(\psi+1)} .
$$

Next let's consider condition (73). This leads to:

$$
\chi_{\pi}>1
$$

Notice that the threshold expressed in condition (75) is negative and thus completely nested in (76).

Proof Corollary 5 Proof. Proposition 4 identifies three relevant conditions. We first have to compute the points where conditions (34) and (35) cross condition (33). It can easily be confirmed that condition (35) crosses condition (33) at:

$$
\psi=\frac{-1-\beta-\kappa}{\kappa}
$$

and that condition (34) crosses condition (33) at:

$$
\psi=\frac{1-\beta-\kappa}{\kappa} .
$$


Notice that (77) is always less than -1 and lies on the left of (78). Moreover, as (35) is increasing in $\psi$ it will always constitute an upper bound to the maximum $\chi_{\pi}$ from $\psi=-1$ up to the point where the conditions (34) and (35) cross each other:

$$
\psi=\frac{(1-\beta)(\sigma+\eta)}{4 \sigma+\kappa(\sigma+\eta)}-1
$$

It also has to be confirmed that point (79) is on the left hand side of the asymptote of condition (34), namely $\frac{\eta-\sigma}{2 \sigma}$. After some tedious algebra it can be proved that this is always the case (under the parameter restrictions outlined). From $\psi$ equal to (79) condition (34) binds from above until the point where it crosses condition (33), at (78). From this point onwards determinacy can never be attained. 